MATHEMATICS OF COMPUTATION

Volume 72, Number 243, Pages 1251-1279

S 0025-5718(03)01492-3

Article electronically published on January 8, 2003

\title{
ON THE CONVERGENCE OF ENTROPY CONSISTENT SCHEMES FOR LUBRICATION TYPE EQUATIONS IN MULTIPLE SPACE DIMENSIONS
}

\author{
GÜNTHER GRÜN
}

\begin{abstract}
We present nonnegativity-preserving finite element schemes for a general class of thin film equations in multiple space dimensions. The equations are fourth order degenerate parabolic, and may contain singular terms of second order which are to model van der Waals interactions. A subtle discretization of the arising nonlinearities allows us to prove discrete counterparts of the essential estimates found in the continuous setting. By use of the entropy estimate, strong convergence results for discrete solutions are obtained. In particular, the limit of discrete fluxes $M_{h}\left(U_{h}\right) \nabla P_{h}$ will be identified with the flux $\mathcal{M}(u) \nabla\left(W^{\prime}(u)-\Delta u\right)$ in the continuous setting. As a by-product, first results on existence and positivity almost everywhere of solutions to equations with singular lower order terms can be established in the continuous setting.
\end{abstract}

\section{INTRODUCTION}

Lubrication approximation allows one to describe the evolution of thin films of viscous liquids on plain surfaces by a fourth order degenerate parabolic equation of the form

$$
u_{t}+\operatorname{div}\left(\mathcal{M}(u) \nabla\left(\Delta u-W^{\prime}(u)\right)\right)=0 \quad \text { in } \Omega \times(0, T) \subset \mathbb{R}^{d+1} .
$$

The film height is denoted by $u$, and the generalized pressure $p:=-\Delta u+W^{\prime}(u)$ is given as the sum of a capillarity term and an additional nonlinearity $W^{\prime}(u)$ which is to model molecular interactions (e.g., van der Waals forces) or effects of gravity. In many applications, $W^{\prime}(\cdot)$ is singular at zero.

The explicit form of the nonlinear mobility $\mathcal{M}(u)$ depends on the boundary condition at the liquid-solid interface. A no-slip condition entails $\mathcal{M}(u)=u^{3}$; various slip conditions (cf. [6] ) yield $\mathcal{M}(u)=u^{3}+\beta u^{n}, \beta>0, n \in(0,3)$. Combined with no-flux boundary conditions and nonnegative initial data, (1) is an initialboundary-value problem which has a number of interesting features.

One peculiarity is the property to admit globally nonnegative solutions. In the simplest case - that of the model problem with $W^{\prime} \equiv 0$ - this follows from the

Received by the editor August 14, 2000 and, in revised form, September 21, 2001.

2000 Mathematics Subject Classification. Primary 35K35, 35K55, 35K65, 35R35, 65M12, 65M60, 76D08.

Key words and phrases. Lubrication approximation, fourth order degenerate parabolic equations, nonnegativity preserving, finite elements. 
so-called entropy estimate

$$
\int_{\Omega} \int_{1}^{u(T, x)} \int_{1}^{s} \frac{1}{\mathcal{M}(r)} d r d s d x+\int_{\Omega_{T}}|\Delta u|^{2}=\int_{\Omega} \int_{1}^{u_{0}(x)} \int_{1}^{s} \frac{1}{\mathcal{M}(r)} d r d s d x .
$$

That estimate goes back to the fundamental work of Bernis and Friedman [8], which gave the first results on existence and nonnegativity of solutions to equation (1) with $W^{\prime} \equiv 0$ in space dimension $d=1$ (for corresponding results in higher space dimensions, we refer to [16] and [14]).

Later on, more refined versions of the entropy estimate could be established (see [5] and [9] in one space dimension, 12 in multiple dimensions). These are key ingredients to prove stronger regularity results as well as results on finite speed of propagation (cf. [7] in one space dimension, [10] in multiple space dimensions) or on a waiting time phenomenon (cf. [13]).

Moreover, if the mobility $\mathcal{M}(\cdot)$ is sufficiently smooth and vanishes at zero, then results on strict positivity follow. In this context, it is worthwhile to introduce the mobility growth exponent $n:=\sup \left\{s \in \mathbb{R}^{+}: \lim _{u \rightarrow 0} \frac{\mathcal{M}(u)}{u^{s}}<\infty\right\}$, which governs the qualitative behaviour of solutions. For instance, if $n>\frac{3}{2}$, then $\operatorname{supp}\left(u\left(t_{1}, \cdot\right)\right) \subset$ $\operatorname{supp}\left(u\left(t_{2}, \cdot\right)\right)$ for $t_{1}<t_{2}$ (see [5], 9], and [12]).

In order to guarantee nonnegativity or even strict positivity of discrete solutions, it seems therefore to be a natural approach to formulate a numerical scheme in such a way that a discrete counterpart of the entropy estimate might be derived. For the model problem with $W^{\prime} \equiv 0$, this was accomplished simultaneously by Zhornitskaya and Bertozzi in [23] and by Rumpf and the author of this paper in [18].

Assuming $\mathcal{M}(\cdot)$ to be of class $C^{2}(\mathbb{R})$, i.e., $n \geq 2$, Zhornitskaya and Bertozzi suggested a time-continuous, space-discrete finite difference scheme for the approximation of strictly positive solutions. For this scheme, they proved strong convergence of positive discrete solutions and showed equivalence to a finite element approach with bilinear elements on uniform, rectangular grids. On account of numerical experiments performed in one space dimension they conjectured that positivitypreserving schemes might also be used to approximate solutions to initial data with compact support.

In [18] Grün and Rumpf considered finite volume - finite element schemes for continuous diffusion coefficients $\mathcal{M}(\cdot)$ (i.e., $n>0$ ) on simplicial grids. In arbitrary space dimensions, results on nonnegativity (even on positivity, if $n>2$ ) of discrete solutions follow and the numerical cost merely consists of solving in each time step a linear system involving a sparse matrix. A time-step control based on an explicit formula for the normal velocity of the free boundary allows one to trace its propagation very precisely. In space dimension $d=1$ the convergence of discrete solutions to a continuous solution in the sense of Theorem 3.1 in [8] could be proven; in higher space dimensions it was only possible to establish a weaker result which in particular did not allow us to identify the limit of the discrete fluxes $J_{h}=M\left(U_{h}\right) \nabla P_{h}$ with the continuous flux $-\mathcal{M}(u) \nabla \Delta u$.

In 17] this algorithmic approach was generalized to equation (1) with nonvanishing nonlinearities $W^{\prime}$, and in special cases results on existence of discrete solutions and on their stability could be established. The publication [19] did not only provide strong convergence results for discrete solutions to equation (11) in space dimension $d=1$; in addition, it contained numerical simulations on dewetting which are in good agreement with physical experiments. 
In the multidimensional case, additional difficulties arise due to the fact that even in the continuous setting no results about boundedness or continuity of solutions are known. With the present paper, we intend to overcome those difficulties; the purpose of the paper is threefold. First, we prove convergence of discrete solutions to the numerical scheme suggested in [17] in multiple space dimensions for a wide class of physically relevant conjoining or disjoining pressure functions $W^{\prime}$ (for details, see section 2). In particular, it will be possible to identify the limit of the discrete fluxes $J_{h}=M\left(U_{h}\right) \nabla P_{h}$ with the continuous flux $\mathcal{M}(u) \nabla\left(W^{\prime}(u)-\Delta u\right)$. As a by-product, we obtain a first existence result in the case that $W^{\prime}(\cdot)$ is singular. Of course, our technique also applies to the case that $W^{\prime} \equiv 0$ and $\mathcal{M}(\cdot)$ is not necessarily bounded. Hence, the convergence results presented in [18] are strongly improved.

It is worth mentioning that equation (1) has a number of common features with the Cahn-Hilliard equation with degenerate mobility, which models phase separation of binary alloys (cf. [14]). In particular, it is not difficult to modify the numerical approach presented in this paper to formulate entropy-consistent schemes for that equation. Hence, the physically relevant space dimensions to be considered in the following are $d=2$ and $d=3$.

Historically, the first successful attempt to construct a finite element scheme guaranteeing nonnegativity of solutions was done by Barrett, Blowey and Garcke (cf. [1] for the case $W^{\prime} \equiv 0$ and the subsequent paper [2] in the context of the CahnHilliard equation). By solving in each time-step an elliptic variational inequality of second order, they force solutions to stay nonnegative. In one space dimension, they succeed in proving convergence to a solution in the sense of Theorem 3.1 in 8]. That solution is not necessarily as regular as the limit of discrete solutions obtained in [18], since a discrete Laplacian of discrete solutions is not controlled.

Let us briefly describe our technique to prove the aforementioned convergence results for numerical schemes in multiple space dimensions. The starting point is a discrete version of the entropy estimate valid also in the case $W^{\prime} \not \equiv 0$ (cf. Lemma 4.3). This estimate provides a uniform boundedness result in $L^{2}\left(\Omega_{T}\right)$ for a certain discrete Laplacian of discrete solutions $U_{h}$. This property enables us to prove that discrete solutions $\left(U_{h}\right)_{h \rightarrow 0}$ are uniformly bounded in $L^{2}\left((0, T) ; C^{\beta}(\Omega)\right)$ for $\beta<2-\frac{d}{2}$, and that their gradients $\left(\nabla U_{h}\right)_{h \rightarrow 0}$ are uniformly bounded in $L^{2}\left((0, T) ; L^{p}(\Omega)\right)$ for $p<\frac{2 d}{d-2}$. The latter result is needed for compactness in time of discrete solutions when $\mathcal{M}(\cdot)$ is not bounded. The former result is a main ingredient to identify the limit of the flux terms $J_{h}$ with $\mathcal{M}(u) \nabla\left(W^{\prime}(u)-\Delta u\right)$.

The outline of the paper is as follows. In section 2 , the initial-boundary value problem will be formulated in the continuous setting, and the physical background of certain growth assumptions will be explained. Section 3 is devoted to algorithmic questions. We formulate the numerical scheme and provide a preliminary existence result for discrete solutions. In section 4, the energy and the entropy estimate follow. These are used in section 5 to prove nonnegativity or positivity results for discrete solutions. Section 6 is one of the main sections of this paper. It contains the aforementioned improved regularity result for discrete solutions. This allows us to prove in section 7 a result on compactness in time in the case that $\mathcal{M}(\cdot)$ is not bounded. Finally, section 8 is devoted to the proof of our convergence/existence result. It also contains a theorem on positivity almost everywhere of solutions in the continuous setting in the case that $W^{\prime}(\cdot)$ is singular. An appendix contains a first numerical example to underline the practicality of the numerical method 
studied in this paper. For more results, in particular on the comparison between numerical simulations and physical experiments, we refer to the joint paper [4] with Becker, Blossey, Jacobs, Mecke, et al. Note that this work shows for the first time that lubrication models are capable of describing thin film dewetting in precise quantitative agreement with experiment over a time-interval that exceeds the initial rupture event by far.

\section{Formulation of the Problem in the CONTINUOUS SETting}

We consider the fourth order degenerate parabolic equation

$$
\begin{aligned}
u_{t}+\operatorname{div}\left(\mathcal{M}(u) \nabla\left(\Delta u-W^{\prime}(u)\right)\right) & =0 & & \text { in } \Omega \times(0, T) \subset \mathbb{R}^{d+1}, \\
\frac{\partial}{\partial \nu} u=\frac{\partial}{\partial \nu} \Delta u & =0 & & \text { on } \partial \Omega \times(0, T), \\
u(0, \cdot) & =u_{0}(\cdot) & & \text { in } \Omega .
\end{aligned}
$$

on a convex polygonal domain $\Omega$ in $\mathbb{R}^{d}$ with $d \in\{2,3\}$. For applications to the thin film equation, the relevant dimension is $d=2$; for applications to the Cahn-Hilliard equation (cf. [1]) $d=3$ becomes important as well.

For ease of presentation, let us assume that $\mathcal{M}(\cdot)$ satisfies

(M1) $\mathcal{M}(s)=(s)_{+}^{n}$ with an arbitrary exponent $n \in \begin{cases}(0, \infty) & \text { if } d \leq 2, \\ (0,4) & \text { if } d=3 .\end{cases}$

Here,

$$
(s)_{+}:= \begin{cases}s & \text { if } s \geq 0, \\ 0 & \text { if } s<0 .\end{cases}
$$

Assume further that the interfacial energy $W \in C^{2}\left(\mathbb{R}^{+} ; \mathbb{R}\right)$ can be decomposed into a sum $W=W_{+}+W_{-}$with a convex, nonnegative function $W_{+} \in C^{2}\left(\mathbb{R}^{+}\right)$and a concave function $W_{-} \in C^{2}\left(\mathbb{R}^{+}\right)$.

To avoid further technical difficulties, let us suppose that $W$ satisfies one of the following hypotheses:

(H1) $W^{\prime}$ is given by $W^{\prime}(s):=W_{+}^{\prime}(s)+W_{-}^{\prime}(s):=-H_{1} s^{-\varrho_{1}}+H_{2} s^{-\varrho_{2}}$ with nonnegative constants $H_{1}, H_{2}$ and positive numbers $\varrho_{1}>\varrho_{2}$ satisfying $\varrho_{1}>$ $2 \varrho_{2}+1>0$ and $H_{1}>0$ if $H_{2}>0$.

(H2) A positive constant $K_{2}$ exists such that $W_{-}^{\prime \prime} \geq-K_{2}$ on $\mathbb{R}$.

Let us make a few comments on the physical background of these hypotheses. If the motion is driven solely by surface tension, then $W^{\prime}$ has to be chosen zero. But in many cases of interest, long range attractive or repulsive forces acting between solid and gas cannot be neglected. A typical example is given by van der Waals forces, which entail an interfacial energy of the form $W(s)=A s^{-2}$. For positive $A$ spreading is enforced, for negative $A$ the corresponding forces are destabilizing. In the latter case wellposedness globally in time can be guaranteed, if certain short range repulsive forces (for instance, effects of Born repulsion) are taken into account. A typical example is given by the classical $6-12$-Lennard-Jones potential, which entails growth assumption (H1) with $\varrho_{1}=9$ and $\varrho_{2}=3$.

On the other hand, gravity effects are modeled by energy functions satisfying (H2). For more information on the physical background, we refer to [21] and the references therein. 


\section{Formulation of the Algorithm-Existence of Discrete solutions}

Let $\mathcal{T}_{h}$ be a regular and admissible triangulation of the domain $\Omega$ (cf. Ciarlet's monograph [11]) with simplicial elements. Let us suppose in addition that the discretization is rectangular, in the sense that

(T1) for each simplicial element $E \in \mathcal{T}_{h}$, a vertex $x_{0}(E)$ exists such that the edges connecting $x_{0}(E)$ with vertices $x_{i}(E)$ and $x_{j}(E)$ are perpendicular to each other for $i, j \in\{1, \cdots, d\}, i \neq j$.

We will take advantage of (T1) mainly in the proof of the entropy estimate (cf. Lemma 4.3). Note that (T1) does not exclude the applicability of standard strategies for local mesh refinement. However, (T1) induces a slight restriction on the class of domains that can be considered. Fortunately, that restriction is negligible in applications. Indeed, we are mainly interested in the spreading of droplets (i.e., compactly supported initial data) or in instabilities of extended films. In both cases, $\Omega$ serves only as a substitute for $\mathbb{R}^{d}$. Therefore, the "fine structure" of $\partial \Omega$ seems to be of minor importance. Hence, in the sequel, when we call $\Omega$ a polygonal domain, we implicitly assume that $\Omega$ permits triangulations $\mathcal{T}_{h}$ endowed with the property $(\mathrm{T} 1)$.

By $V^{h}$, we denote the subspace of $H^{1,2}(\Omega)$ consisting of continuous functions which are linear on each element $E \in \mathcal{T}_{h}$. In the following, elements of $V^{h}$ will be denoted by capitals, and functions contained in nondiscrete function spaces will be denoted by lower-case characters. A function $V \in V^{h}$ is uniquely defined by its values on the set of nodes $\mathcal{N}_{h}=\left\{x_{j}\right\}_{j \in J}$ of the triangulation $\mathcal{T}_{h}$, where $J$ denotes a corresponding index set. A set of basis functions dual to the set of nodal points $\mathcal{N}_{h}$ is given by the "hat"-type functions $\varphi_{j} \in V^{h}$ with $\varphi_{j}\left(x_{i}\right)=\delta_{i j}$. Let us furthermore introduce the well-known lumped masses scalar product corresponding to the integration formula

$$
(\Theta, \Psi)_{h}:=\int_{\Omega} \mathcal{I}_{h}(\Theta \Psi)
$$

where $\mathcal{I}_{h}: C^{0}(\Omega) \rightarrow V^{h}$ is the interpolation operator with $\mathcal{I}_{h} u=\sum_{j \in J} u\left(x_{j}\right) \varphi_{j}$.

By $(u, v)$, we denote the usual $L^{2}$-scalar product on $\Omega$. The diagonal, positive definite lumped masses matrix is given by $\left(M_{h}\right)_{i j}=\left(\varphi_{i}, \varphi_{j}\right)_{h}$, and $L_{h}$ stands for the standard stiffness matrix $\left(L_{h}\right)_{i j}=\left(\nabla \varphi_{i}, \nabla \varphi_{j}\right)$. The canonical basis on $\mathbb{R}^{d}$ is denoted by $\left\langle e_{1}, \cdots, e_{d}\right\rangle$. Let the time interval $[0, T]$ be subdivided into intervals $I_{k}=\left[t_{k}, t_{k+1}\right)$ with $t_{k+1}=t_{k}+\tau_{k}$ for time increments $\tau_{k}>0$ and $k=0, \cdots, N-1$. For simplicity, we assume $\tau_{k} \equiv \tau$ for $k=0, \cdots, N-1$. We will denote the backward difference quotients with respect to time by $\partial_{\tau}^{-}$. Finally, we introduce $S^{0,-1}\left(V^{h}\right)$ as the space of functions $v:[0, T] \rightarrow V^{h}$ which are constant on each $I_{k}, k=$ $0, \cdots, N-1$.

We recall the following well-known estimates:

$$
\left|(U, V)-(U, V)_{h}\right| \leq C h^{1+l}\|U\|_{l}\|V\|_{1} \quad \text { for all } U, V \in V_{h}, \quad l=0,1 .
$$

In the same spirit, there exist positive constants $c, C$ such that for $|\cdot|_{h}:=\sqrt{(., .)_{h}}$ we have

$$
c|\cdot|_{h}^{2} \leq(., .) \leq C|\cdot|_{h}^{2} .
$$

Then, an implicit, backward Euler discretization scheme for equation (2) reads as follows: 
For given $U^{0} \in V^{h}$ and $k=0, \cdots, N-1$, find functions $\left(U^{k+1}, P^{k+1}\right) \in V^{h} \times V^{h}$ such that

$$
\begin{aligned}
\left(\partial_{\tau}^{-} U^{k+1}, \Theta\right)_{h}+\left(M\left(U^{k+1}\right) \nabla P^{k+1}, \nabla \Theta\right) & =0 \\
\left(\nabla U^{k+1}, \nabla \Psi\right)+\left(W_{+}^{\prime}\left(U^{k+1}\right), \Psi\right)_{h}+\left(W_{-}^{\prime}\left(U^{k}\right), \Psi\right)_{h} & =\left(P^{k+1}, \Psi\right)_{h}
\end{aligned}
$$

for all $\Theta, \Psi \in V^{h}$.

Note that only the destabilizing term $W_{-}^{\prime}$ is discretized explicitly - a strategy which is reminiscent of a method used for the discretization of nonlinear source terms in second order semilinear parabolic equations (cf. [15]). Later on, this will entail in a very natural way estimates controlling the total energy at a given time $t>0$ in terms of the initial energy. However, for the discretization of fourth order degenerate parabolic equations we need additional concepts which are rather subtle and which go far beyond the strategies applied in the case of second order equations. This is mainly due to the fact that these degenerate equations admit globally nonnegativity-preserving solutions - a property which is rather peculiar in the class of fourth order parabolic equations. Heuristically, this particular behavior occurs since the mobility $\mathcal{M}(\cdot)$ degenerates at zero, and therefore the flux $J=$ $\mathcal{M}(u) \nabla p$ vanishes as soon as $u$ approaches zero. As a consequence, the crucial point, both with respect to analytical results on nonnegativity, positivity and convergence of discrete solutions as well as to the performance of the algorithm, is the way the mobility $\mathcal{M}(\cdot)$ is discretized. In previous works (18], [19], 23]; see also [3] for an application to Cahn-Hilliard systems), it was shown that a certain class of harmonic integral means is an efficient ansatz to accomplish this. Moreover, in the multi-dimensional case it will be necessary to replace the scalar-valued mobility $\mathcal{M}(\cdot)$ in the discrete setting by a matrix-valued mobility field $M$ in order to have optimal control of different flux components. So let us introduce the notion of an admissible entropy-mobility pair, which was proposed in [18].

Let $m: \mathbb{R} \rightarrow \mathbb{R}_{0}^{+}$be an approximation of the continuous mobility $\mathcal{M}($.$) , and take$ $A$ as a fixed nonnegative number - both will be specified later on. We call a pair of functions $G: \mathbb{R} \rightarrow \mathbb{R}_{0}^{+}, M: V^{h} \rightarrow \bigotimes_{k=1}^{\left|\mathcal{T}_{h}\right|} \mathbb{R}^{d \times d}$ an admissible entropy-mobility pair with respect to the triangulation $\mathcal{T}_{h}$ if the following axioms are satisfied:

(i) $M: V^{h} \rightarrow \bigotimes_{k=1}^{\left|\mathcal{T}_{h}\right|} \mathbb{R}^{d \times d}$ is continuous,

(ii) $\left.M(U)\right|_{E}=m(U)$ Id if $\left.U\right|_{E}$ is constant,

(iii) $M(U) \nabla \mathcal{I}_{h} G^{\prime}(U)=\nabla U$, where $G(s):=\int_{A}^{s} g(r) d r$ with $g(s)=\int_{A}^{s} m(r)^{-1} d r$, and

(iv) on each element $E$, the matrix $\left.M(U)\right|_{E}$ is symmetric and positive semidefinite.

By construction, $G$ is nonnegative and convex. For nondegenerate reference simplices $\hat{E}_{\left(\alpha_{1}, \cdots, \alpha_{d}\right)}:=\operatorname{co}\left(0, \alpha_{1} e_{1}, \cdots, \alpha_{d} e_{d}\right)$, the axioms above are satisfied by

$$
\begin{aligned}
\hat{M}= & \left(\varrho\left(U(0), U\left(\alpha_{i} e_{i}\right)\right) \delta_{i j}\right)_{i, j=1, \cdots, d} \\
& \text { with } \varrho(x, y):=\left(f_{x}^{y} \frac{1}{m(s)} d s\right)^{-1} \delta_{i j} .
\end{aligned}
$$

Note that for $U\left(\alpha_{k} e_{k}\right)=U(0)$ the definition simplifies to $\hat{M}_{k k}=m(U(0))$. For elements $E$ which can be mapped onto a reference element $\hat{E}_{\left(\alpha_{1}, \cdots, \alpha_{d}\right)}$ by an orthogonally affine equivalent transformation $x \mapsto \hat{x}=x_{0}+A^{-1} x, A$ an orthogonal matrix, the mobility matrix is given by $M:=A \hat{M} A^{-1}$ (for details, cf. [18]). 
To proceed, we will need explicit formulae for the mobility matrices corresponding to $\mathcal{M}(s)=(s)_{+}^{n}$. In order to optimize nonnegativity properties of discrete solutions, we will distinguish two cases (for details the reader is referred to [18]). If $n \geq 1$, we take for positive $\sigma \ll 1$ the function $m_{\sigma}(s)=\mathcal{M}(\max (\sigma, s))$ as approximation to the continuous mobility $\mathcal{M}(\cdot)$. For $\varrho_{\sigma}(\cdot, \cdot)$, we obtain

$$
\varrho_{\sigma}\left(U_{1}, U_{0}\right)= \begin{cases}(n-1) \frac{U_{1}-U_{0}}{U_{0}^{1-n}-U_{1}^{1-n}} & \text { if } U_{0}, U_{1}>\sigma, \quad U_{0} \neq U_{1}, \\ \frac{(n-1)\left(U_{1}-U_{0}\right)}{\sigma^{1-n}-U_{1}^{1-n}+(1-n)\left(U_{0}-\sigma\right) \sigma^{-n}} & \text { if } U_{1} \geq \sigma, \quad U_{0} \leq \sigma, \quad U_{1} \neq U_{0}, \\ \sigma^{n} & \text { if } U_{1}, U_{0} \leq \sigma \\ U_{0}^{n} & \text { if } U_{1}=U_{0}>\sigma,\end{cases}
$$

if $n>1$ and

$$
\varrho_{\sigma}\left(U_{1}, U_{0}\right)= \begin{cases}\frac{U_{1}-U_{0}}{\log \left(U_{1}\right)-\log \left(U_{0}\right)} & \text { if } U_{0}, U_{1}>\sigma, \quad U_{0} \neq U_{1}, \\ \frac{U_{1}-U_{0}}{\log \left(U_{1}\right)-\log (\sigma)-\left(U_{0}-\sigma\right) \sigma^{-1}} & \text { if } U_{1} \geq \sigma, \quad U_{0} \leq \sigma, \quad U_{1} \neq U_{0}, \\ \sigma & \text { if } U_{1}, U_{0} \leq \sigma, \\ U_{0} & \text { if } U_{1}=U_{0}>\sigma,\end{cases}
$$

if $n=1$.

If $0<n<1$, we define the discrete entropy $G_{\sigma}$ for $0<\sigma \ll 1$ as

$$
G_{\sigma}(s):= \begin{cases}\frac{1}{(1-n)(2-n)} s^{2-n} & \text { if } s \geq 0 \\ \frac{1}{\sigma} \frac{1}{(1-n)(2-n)}(-s)^{2-n} & \text { if } s<0 .\end{cases}
$$

We take

$$
m_{\sigma}(s):= \begin{cases}\left(G_{\sigma}^{\prime \prime}(s)\right)^{-1} & \text { if } s \neq 0 \\ 0 & \text { if } s=0\end{cases}
$$

and $A \equiv 0$. Hence, for $\varrho_{\sigma}(\cdot, \cdot)$ we obtain

$$
\varrho_{\sigma}\left(U_{1}, U_{0}\right):= \begin{cases}\frac{(1-n)\left(U_{1}-U_{0}\right)}{U_{1}^{1-n}-U_{0}^{1-n}} & \text { if } \min \left(U_{1}, U_{0}\right) \geq 0, U_{1} \neq U_{0}, \\ \frac{(1-n)\left(U_{1}-U_{0}\right)}{\sigma^{-1}\left|U_{0}\right|^{1-n}+U_{1}^{1-n}} & \text { if } U_{0}<0<U_{1}, \\ \sigma \frac{(n-1)\left(U_{1}-U_{0}\right)}{\left|U_{0}\right|^{1-n}-\left|U_{1}\right|^{1-n}} & \text { if } \max \left(U_{1}, U_{0}\right) \leq 0, U_{1} \neq U_{0}, \\ \mathcal{M}\left(U_{1}\right) & \text { if } U_{1}=U_{0} \geq 0, \\ \sigma \mathcal{M}\left(-U_{1}\right) & \text { if } U_{1}=U_{0}<0 .\end{cases}
$$

For the reader's convenience, let us give at least a few additional comments on the meaning of the parameter $0<\sigma<1$. If $n \geq 1, \sigma$ serves as a regularization parameter which guarantees that $\varrho_{\sigma}(s, t)$ is well defined also for arguments $s, t \leq 0$. In contrast, if $0<n<1, \sigma$ plays the role of a penalization parameter that is used to enforce nonnegativity of discrete solutions. Indeed, we will prove in section 4 a kind of $L^{\infty}\left(I ; L^{1}(\Omega)\right)$-estimate for $G_{\sigma}(U)$. Combining this result with equation (9), it becomes evident that the smaller $\sigma$, the more strongly negative values of discrete solutions will be penalized. Finally, let us emphasize once more that there are not only technical reasons, e.g., the availability of the entropy estimate and thus of improved results on regularity, which are in favor of our strategy of harmonic integral averages. Unpublished numerical experiments indicate that for more naive approaches, using for instance arithmetic averages, the performance of the algorithm in fact drastically deteriorates with respect to preservation of nonnegativity. 
In the sequel, we will need the following estimate on the integrability of the field $M_{\sigma}(\cdot)$ of discrete mobility matrices. To avoid clumsy notation, for given $U \in V^{h}$ we will use the abbreviation $M(U)$ for the function $\hat{M}(U): \Omega \rightarrow \mathbb{R}^{d \times d}$ that satisfies for $x \in E$ the relation $\hat{M}(U)(x):=\left.M(U)\right|_{E}$. The lemma reads as follows.

Lemma 3.1. Let $\mathcal{M}(s):=(s)_{+}^{n}$ and assume that for $0<\sigma<1$ the field of mobility matrices $M_{\sigma}: V^{h} \rightarrow \bigotimes_{k=1}^{\left|\mathcal{T}_{h}\right|} \mathbb{R}^{d \times d}$ is given as above. Then, a positive constant $C$ exists, which only depends on the regularity of the mesh, such that for all $U \in V^{h}$ we have

$$
\left|\int_{\Omega} M_{\sigma}(U) d x\right| \leq C\left(\int_{\Omega}|U|^{n} d x+1\right) .
$$

Proof. Since $M_{\sigma}(U)$ is symmetric, positive semidefinite and constant on each element $E \in \mathcal{T}_{h}$, it is sufficient to estimate its eigenvalues, i.e., to estimate $\varrho_{\sigma}\left(U_{i}, U_{0}\right)$ (cf. (7)-(8) $), i=1, \cdots, d$. Let us first show the existence of a positive constant $C$ such that for all $0<\sigma<1$

$$
\varrho_{\sigma}\left(U_{1}, U_{0}\right) \leq C\left(\left|U_{1}\right|^{n}+\left|U_{0}\right|^{n}+1\right) .
$$

We first consider the case $n \geq 1$. Observing that $G_{\sigma}: \mathbb{R} \rightarrow \mathbb{R}_{0}^{+}$is of class $C^{2}$ and that $\varrho_{\sigma}\left(U_{1}, U_{0}\right)$ can be rewritten as

$$
\varrho_{\sigma}\left(U_{1}, U_{0}\right)=\left(\frac{G_{\sigma}^{\prime}\left(U_{1}\right)-G_{\sigma}^{\prime}\left(U_{0}\right)}{U_{1}-U_{0}}\right)^{-1},
$$

we infer by means of Taylor's formula the existence of a number $\xi \in\left[U_{0}, U_{1}\right]$ such that $\varrho\left(U_{1}, U_{0}\right)=m_{\sigma}(\xi)$. Hence, if $\min \left(U_{0}, U_{1}\right) \geq \sigma$, we see immediately that $m_{\sigma}(\xi)=\xi^{n} \leq U_{0}^{n}+U_{1}^{n}$. On the other hand, if $U_{1} \geq \sigma$ and $U_{0} \leq \sigma$, then $\sigma^{n} \leq m_{\sigma}(\xi) \leq U_{1}^{n}$. Using for the remaining cases the aforementioned assumption $0<\sigma<1$, the desired estimate (12) can easily be established.

Let us now consider the case $0<n<1$. We will only discuss the subcase $U_{0}<0<U_{1}$, as the other cases are straightforward consequences of Taylor's formula. Observing that

$$
\begin{aligned}
\varrho_{\sigma}\left(U_{1}, U_{0}\right) & \leq \frac{(1-n)\left(U_{1}+\left|U_{0}\right|\right)}{\left|U_{0}\right|^{1-n}+U_{1}^{1-n}} \\
& \leq(1-n)\left(U_{1}^{n}+\left|U_{0}\right|^{n}\right)
\end{aligned}
$$

for arbitrary numbers $U_{1}>0, U_{0}<0$ and $0<\sigma<1$, we see that (12) holds also in the case $0<n<1$. Therefore, on each element $E \in \mathcal{T}_{h}$ we have

$$
\left|M_{\sigma}(U)\right| \leq C\left(\sum_{i=0}^{d}\left|U_{i}(E)\right|^{n}+1\right) .
$$

Now observe that the function $F(U):=\sum_{i=0}^{d}\left|U_{i}\right|^{n}$ is homogeneous of degree $n$ and that it only vanishes for $U_{0}=\cdots=U_{d}=0$. The same obviously also holds for the function $G(U):=f_{E}|U|^{n} d x$. Hence, we infer by homogeneity that

$$
\int_{E} F(U) d x \leq C \int_{E}|U|^{n}
$$

where the constant $C$ depends on the mesh regularity. Summing up,

$$
\int_{\Omega}\left|M_{\sigma}(U)\right| \leq C\left(\int_{\Omega}|U|^{n}+1\right) .
$$


If $W$ is sufficiently regular (for instance, if (H2) holds and $W^{\prime}$ is nonsingular), existence of discrete solutions can be proven on the basis of Brouwer's fixed-point theorem. If (H1) is satisfied, we need additional results on strict positivity, which will be given in section 5 Let us formulate a basic existence result for discrete solutions:

Lemma 3.2. Assume that $W \in C^{1}(\mathbb{R} ; \mathbb{R})$ can be decomposed into a sum $W=$ $W_{+}+W_{-}$with a convex function $W_{+} \in C^{1}\left(\mathbb{R} ; \mathbb{R}_{0}^{+}\right)$and a concave function $W_{-} \in$ $C^{1}(\mathbb{R} ; \mathbb{R})$. For arbitrary initial data $U^{0} \in V^{h}$, a discrete solution $(U, P)$ of (国)-(络) does exist on $[0, T]$.

Remark. Note that we do not require $W$ to be nonnegative. Nor do we impose further growth conditions on $W_{-}$.

Proof. For $Z^{k}=U^{k}-\alpha$ with $\alpha:=\frac{1}{|\Omega|} \int_{\Omega} U^{0}$, we are looking for $Z^{k+1}$ satisfying

$$
\left(\nabla Z^{k+1}, \nabla \Psi\right)+\left(W_{+}^{\prime}\left(Z^{k+1}+\alpha\right), \Psi\right)_{h}+\left(W_{-}^{\prime}\left(Z^{k}+\alpha\right), \Psi\right)_{h}=\left(P^{k+1}, \Psi\right)_{h} .
$$

Denoting the weighted stiffness matrix $L_{h}(Z)$ for $Z \in V^{h}$ by

$$
L_{h}(Z)_{i j}:=\int_{\Omega} M(Z+\alpha) \nabla \varphi_{i} \cdot \nabla \varphi_{j}, \quad i, j \in\left\{1, \cdots, \operatorname{dim} V^{h}\right\},
$$

we have to solve the following nonlinear system of $q=\operatorname{dim} V^{h}$ equations in each time step:

$F(\bar{Z})=\bar{Z}-\bar{Z}^{k}+\tau_{k} M_{h}^{-1} L_{h}(\bar{Z})\left(M_{h}^{-1} L_{h} \bar{Z}+W_{+}^{\prime}(Z+\alpha)+W_{-}^{\prime}\left(Z^{k}+\alpha\right)\right)=0$.

Here, we denoted the nodal value vector for a function $V \in V^{h}$ by $\bar{V}$, and with a slight misuse of notation we rewrote $L_{h}(\bar{Z})$ for $L_{h}(Z)$ and $W^{\prime}(Z+\alpha)$ for $\overline{\mathcal{I}_{h} W^{\prime}(Z+\alpha)}$.

Let us now introduce a new bilinear form on $\mathbb{R}^{q}$ by

$$
\langle\bar{Z}, \bar{V}\rangle:=\left(L_{h} \bar{Z}\right)^{T} \bar{V}
$$

where $(\bar{V})^{T}$ denotes the transpose of a vector $\bar{V} \in \mathbb{R}^{q}$. By definition this form is symmetric and therefore a scalar product on $K^{\perp}:=\left\{\bar{W} \mid\left(M_{h} \bar{W}\right)^{T} \mathbb{1}=0\right\}$, where $\mathbb{1}=(1, \cdots, 1)^{T}$. By $\|$.$\| , we denote \langle., .\rangle^{\frac{1}{2}}$. We easily verify that $\bar{Z}^{0} \in K^{\perp}$, and by induction that $F: K^{\perp} \rightarrow K^{\perp}$.

Let us assume now that, for a positive number $R$ to be specified later on, a root $F(\bar{Z})=0$ did not exist on $B_{R}(0)$. Then, due to Brouwer's fixed-point theorem, the mapping $G: B_{R}(0) \rightarrow B_{R}(0)$ defined by $G(\bar{X}):=-\frac{R F(\bar{X})}{\|F(\bar{X})\|}$ would have a fixedpoint $\bar{X}_{0} \in K^{\perp}$ satisfying $\left\|\bar{X}_{0}\right\|=R$. Let us choose $\bar{V}=\bar{V}_{1}+\bar{V}_{2}$ in such a way that

$$
L_{h} \bar{V}_{1}=M_{h}\left(W_{+}^{\prime}\left(X_{0}+\alpha\right)-\frac{\left(M_{h} W_{+}^{\prime}\left(X_{0}+\alpha\right)\right)^{T} \mathbb{1}}{\mathbb{1}^{T} M_{h} \mathbb{1}} \mathbb{1}\right)
$$

and

$$
L_{h} \bar{V}_{2}=M_{h}\left(W_{+}^{\prime}\left(Z^{k}+\alpha\right)-\frac{\left(M_{h} W_{+}^{\prime}\left(Z^{k}+\alpha\right)\right)^{T} \mathbb{1}}{\mathbb{1}^{T} M_{h} \mathbb{1}} \mathbb{1}\right)
$$


which is possible since $\operatorname{ker}\left(L_{h}\right)=\langle\mathbb{1}\rangle$. Using the relation $X_{0}^{T} M_{h} \mathbb{1}=0$, we may estimate $\left\langle\bar{X}_{0}, \bar{X}_{0}+\bar{V}\right\rangle$ in two different ways:

$$
\begin{aligned}
\left\langle\bar{X}_{0}, \bar{X}_{0}+\bar{V}\right\rangle= & \left\|\bar{X}_{0}\right\|^{2}+\bar{X}_{0}^{T} M_{h} W_{+}^{\prime}\left(X_{0}+\alpha\right)+\bar{X}_{0}^{T} M_{h} W_{-}^{\prime}\left(Z^{k}+\alpha\right) \\
= & \left\|\bar{X}_{0}\right\|^{2}+\left(\bar{X}_{0}+\alpha \mathbb{1}-\alpha \mathbb{1}\right)^{T} M_{h}\left(W_{+}^{\prime}\left(X_{0}+\alpha\right)-W_{+}^{\prime}(\alpha) \mathbb{1}\right) \\
& \quad+\bar{X}_{0}^{T} M_{h} W_{-}^{\prime}\left(Z^{k}+\alpha\right)+W_{+}^{\prime}(\alpha) X_{0}^{T} M_{h} \mathbb{1}
\end{aligned}
$$

Here, we used the monotonicity of $W_{+}^{\prime}$ as well as the fact that $M_{h}$ is a diagonal matrix. Taking into account the equivalence of norms on finite dimensional spaces, we may find $R_{1}>0$ such that

$$
\left\langle\bar{X}_{0}, \bar{X}_{0}+\bar{V}\right\rangle>0
$$

if $\left\|\bar{X}_{0}\right\|=R$ and $R \geq R_{1}$.

On the other hand, by convexity of $W_{+}$we obtain (recall: $F\left(\bar{X}_{0}\right) \in K^{\perp}$ !)

$$
\begin{aligned}
\left\langle F\left(\bar{X}_{0}\right)\right. & \left., \bar{X}_{0}+\bar{V}\right\rangle \\
= & \left\langle\bar{X}_{0}-\bar{Z}^{k}, \bar{X}_{0}\right\rangle+\left(\bar{X}_{0}-\bar{Z}^{k}\right)^{T} M_{h}\left(W_{+}^{\prime}\left(X_{0}+\alpha\right)+W_{-}^{\prime}\left(Z^{k}+\alpha\right)\right) \\
& +\tau\left(L_{h}\left(\bar{X}_{0}\right)\left(M_{h}^{-1} L_{h} \bar{X}_{0}+W_{+}^{\prime}\left(X_{0}+\alpha\right)+W_{-}^{\prime}\left(Z^{k}+\alpha\right)\right)\right)^{T} \\
& *\left(M_{h}^{-1} L_{h} \bar{X}_{0}+W_{+}^{\prime}\left(X_{0}+\alpha\right)+W_{-}^{\prime}\left(Z^{k}+\alpha\right)\right) \\
\geq & \frac{1}{2}\left\|\bar{X}_{0}\right\|^{2}-\frac{1}{2}\left\|\bar{Z}^{k}\right\|^{2}+\left(\bar{X}_{0}-\bar{Z}^{k}\right)^{T} M_{h} W_{+}^{\prime}\left(X_{0}+\alpha\right) \\
& +\left(\bar{X}_{0}-\bar{Z}^{k}\right)^{T} M_{h} W_{-}^{\prime}\left(Z^{k}+\alpha\right) \\
\geq & \frac{1}{4}\left\|\bar{X}_{0}\right\|^{2}-\frac{1}{2}\left\|\bar{Z}^{k}\right\|^{2}+\left(\mathbb{1}, W_{+}\left(X_{0}+\alpha\right)-W_{+}\left(Z^{k}+\alpha\right)\right)_{h} \\
& -C \cdot\left(W_{-}^{\prime}\left(Z^{k}+\alpha\right)\right)^{T} M_{h} W_{-}^{\prime}\left(Z^{k}+\alpha\right) \\
& -\left(\bar{Z}^{k}\right)^{T} M_{h} W_{-}^{\prime}\left(Z_{k}+\alpha\right) \\
> & 0
\end{aligned}
$$

if $\left\|\bar{X}_{0}\right\|>R \geq R_{2}$ for sufficiently large $R_{2}$. Here, $C$ is a positive constant which only depends on $\mathcal{T}_{h}$.

Hence, $\left\langle\bar{X}_{0}, \bar{X}_{0}+\bar{V}\right\rangle=-\frac{R}{\left\|F\left(\bar{X}_{0}\right)\right\|}\left\langle F\left(\bar{X}_{0}\right), \bar{X}_{0}+\bar{V}\right\rangle<0$ if $R \geq \max \left\{R_{1}, R_{2}\right\}$, a contradiction to (16). This proves the existence of discrete solutions.

\section{Basic a PRIORI estimates-CompaCtness in SPACE}

In this section, we provide the integral estimates necessary for results on compactness with respect to space. We begin with 
Lemma 4.1 (Energy estimate). Let $(U, P) \in S^{0,-1}\left(V^{h}\right) \times S^{0,-1}\left(V^{h}\right)$ be a discrete solution of (5) and (6). Then the following a priori estimate holds:

$$
\begin{aligned}
\frac{1}{2} \int_{\Omega}\left|\nabla U^{N}(x)\right|^{2} d x & +\int_{\Omega} \mathcal{I}_{h} W\left(U^{N}\right) d x+\int_{0}^{T}(M(U) \nabla P, \nabla P) d t \\
& +\frac{\tau}{2} \sum_{i=0}^{N-1} \int_{\Omega}\left|\nabla\left(U^{i+1}(x)-U^{i}(x)\right)\right|^{2} d x \\
& \leq \frac{1}{2} \int_{\Omega}\left|\nabla U^{0}(x)\right|^{2} d x+\int_{\Omega} \mathcal{I}_{h} W\left(U^{0}\right) d x .
\end{aligned}
$$

As the proof is similar to that of Lemma 3.2 in [19], we omit it. But we provide the following corollary.

Corollary 4.2. Let (H1), (H2) and the assumptions of Lemma 4.1 be satisfied. Assume further that

$$
\int_{\Omega}\left|\nabla U_{h}^{0}\right|^{2}+\int_{\Omega} W\left(U_{h}^{0}\right) \leq C<\infty
$$

uniformly, as $h$ tends to zero. Then there exists a positive constant $C(T)$ such that

$$
\begin{aligned}
& \left\|U_{h}\right\|_{L^{\infty}\left(I ; H^{1,2}(\Omega)\right)}+\left\|\mathcal{I}_{h} W\left(U_{h}\right)\right\|_{L^{\infty}\left(I ; L^{1}(\Omega)\right)} \\
& \quad+\left\|\left(M\left(U_{h}\right) \nabla P_{h}, \nabla P_{h}\right)\right\|_{L^{1}((0, T))} \leq C(T) .
\end{aligned}
$$

If (H1) holds, then $C$ is independent of time.

Proof. If (H2) holds, the assertion follows by a combination of Poincaré's inequality and a discrete version of Gronwall's lemma (cf. [15]). For (H1), the result is a straightforward consequence of the boundedness of $W$ from below.

We proceed with the entropy estimate, which will be important for two reasons. First, it yields uniform $L^{2}\left(\Omega_{T}\right)$-integrability of the discrete Laplacian $\Delta_{h} U_{h}$ - the main ingredient in the convergence proof. Second, it is the key for nonnegativity results in case (H2).

The proof of this entropy estimate relies on the property of the triangulation $\mathcal{T}_{h}$ to be rectangular (cf. (T1)). This means, for each element $E_{k} \in \mathcal{T}_{h}, k=1, \cdots,\left|\mathcal{T}_{h}\right|$, there exist a nondegenerate reference simplex $\hat{E}_{\left(\alpha_{1}, \cdots, \alpha_{d}\right)}^{k}$ and an orthogonal matrix $A_{k}$ such that $E_{k} \rightarrow \hat{E}_{\left(\alpha_{1}, \cdots, \alpha_{d}\right)}^{k}$ by the affine mapping $x \rightarrow A_{k}\left(x-x_{0}\left(E_{k}\right)\right)$.

We need some more notation. Given a finite element function $U \in V^{h}$ and an element $E_{k} \in \mathcal{T}_{h}$, we consider the function $\hat{U}_{k}: \hat{E}_{\left(\alpha_{1}, \cdots, \alpha_{d}\right)}^{k} \rightarrow \mathbb{R}$ defined by $\hat{U}_{k}(\hat{x}):=U\left(x_{0}\left(E_{k}\right)+A_{k}^{-1} \hat{x}\right), \quad \hat{x} \in \hat{E}_{\left(\alpha_{1}, \cdots, \alpha_{d}\right)}^{k}$.

In addition, we introduce for $C^{1}$-functions $\varphi: \mathbb{R} \rightarrow \mathbb{R}$ and $U \in V^{h}$ the quantity

$$
\begin{aligned}
& \langle\hat{\nabla} U, \operatorname{diag}(\partial \varphi(U)) \hat{\nabla} U\rangle \\
& \quad:=\sum_{k=1}^{\left|\mathcal{T}_{h}\right|}\left|\hat{E}_{\left(\alpha_{1}, \cdots, \alpha_{d}\right)}^{k}\right| \nabla_{\hat{x}} \hat{U}_{k} \cdot \operatorname{diag}\left(\partial_{1} \varphi\left(\hat{U}_{k}\right), \cdots, \partial_{d} \varphi\left(\hat{U}_{k}\right)\right) \cdot \nabla_{\hat{x}} \hat{U}_{k},
\end{aligned}
$$

where

$i=1, \cdots, d$.

$$
\partial_{i} \varphi\left(\hat{U}_{k}\right):= \begin{cases}\frac{\varphi\left(\hat{U}_{k}\left(\alpha_{i}^{k} e_{i}\right)\right)-\varphi\left(\hat{U}_{k}(0)\right)}{\hat{U}_{k}\left(\alpha_{i}^{k} e_{i}\right)-\hat{U}_{k}(0)} & \text { if } \hat{U}_{k}\left(\alpha_{i}^{k} e_{i}\right) \neq \hat{U}_{k}(0), \\ \varphi^{\prime}\left(\hat{U}_{k}(0)\right) & \text { otherwise }\end{cases}
$$


Lemma 4.3 (Entropy estimate). Let $(U, P)$ be a solution to the system of equations (5) -(6) , let $(M, G)$ be an admissible entropy-mobility pair and let one of the hypotheses (H1), (H2) hold. Assume furthermore that the triangulation $\mathcal{T}_{h}$ satisfies (T1). Moreover, let the solution $U$ be strictly positive if (H2) is not satisfied 1

Then, for arbitrary $T=K \tau, K \in \mathbb{N}$, the following estimate holds:

$$
\begin{aligned}
\int_{\Omega} \mathcal{I}_{h} G(U(T, x)) d x & +\int_{\tau}^{T}\left\|P(t, \cdot)-W_{+}^{\prime}(U(t, \cdot))-W_{-}^{\prime}(U(t-\tau, \cdot))\right\|_{h}^{2} d t \\
& +\frac{1}{2} \int_{\tau}^{T}\left\langle\hat{\nabla} U, \operatorname{diag}\left(\partial W_{+}^{\prime}(U)\right) \hat{\nabla} U\right\rangle \\
\leq \int_{\Omega} \mathcal{I}_{h} G\left(U^{0}(x)\right) d x & +R
\end{aligned}
$$

If (H1) holds, then

$$
R:=\frac{H_{2}^{2}}{H_{1}} C\left(\varrho_{1}, \varrho_{2}\right) \int_{\tau}^{T}\|\nabla U\|^{2}+\sup _{x_{i} \in \mathcal{N}^{h}}\left|W_{-}^{\prime \prime}\left(U^{0}\left(x_{i}\right)\right)\right| \int_{0}^{2 \tau}\|\nabla U\|^{2} .
$$

Otherwise,

$$
R:=K_{2}\left(\int_{0}^{T-\tau}\|\nabla U\|^{2}+\int_{0}^{2 \tau}\|\nabla U\|^{2}\right) .
$$

Remark. Note that $\left|W_{-}^{\prime \prime}\left(U_{h}^{0}(\cdot)\right)\right|$ is uniformly bounded in $h \rightarrow 0$ if $u_{0}$ is strictly positive or if (H2) holds.

Proof. We take the function $\Theta:=\mathcal{I}_{h} G^{\prime}\left(U^{k+1}\right)$ as test function in the weak formulation (5)-(6). Using the convexity of $G(\cdot)$, we obtain

$$
\frac{1}{\tau}\left(\int_{\Omega} \mathcal{I}_{h} G\left(U^{k+1}\right)-\int_{\Omega} \mathcal{I}_{h} G\left(U^{k}\right)\right)+\left(\nabla P^{k+1}, \nabla U^{k+1}\right) \leq 0 .
$$

Relation (6) implies

$$
\begin{aligned}
\left(\nabla P^{k+1}, \nabla U^{k+1}\right)= & \left(P^{k+1}, P^{k+1}\right)_{h}-\left(W_{+}^{\prime}\left(U^{k+1}\right)+W_{-}^{\prime}\left(U^{k}\right), P^{k+1}\right)_{h} \\
= & \left\|P^{k+1}-W_{+}^{\prime}\left(U^{k+1}\right)-W_{-}^{\prime}\left(U^{k}\right)\right\|_{h}^{2}-\left\|W_{+}^{\prime}\left(U^{k+1}\right)+W_{-}^{\prime}\left(U^{k}\right)\right\|_{h}^{2} \\
& \quad+\left(W_{+}^{\prime}\left(U^{k+1}\right)+W_{-}^{\prime}\left(U^{k}\right), P^{k+1}\right)_{h} \\
= & \left\|P^{k+1}-W_{+}^{\prime}\left(U^{k+1}\right)-W_{-}^{\prime}\left(U^{k}\right)\right\|_{h}^{2} \\
& \quad+\left(\nabla U^{k+1}, \nabla \mathcal{I}_{h}\left(W_{+}^{\prime}\left(U^{k+1}\right)+W_{-}^{\prime}\left(U^{k}\right)\right)\right) .
\end{aligned}
$$

Integration with respect to time gives (note: $\int_{0}^{T}=\tau \sum_{k=0}^{N-1}$ )

$$
\begin{aligned}
\int_{\Omega} \mathcal{I}_{h} G(U(T, \cdot)) & +\int_{\tau}^{T}\left\|P(t, \cdot)-W_{+}^{\prime}(U(t, \cdot))-W_{-}^{\prime}(U(t-\tau, \cdot))\right\|_{h}^{2} \\
& \leq \int_{\Omega} \mathcal{I}_{h} G\left(U^{0}(\cdot)\right)-\tau \sum_{k=0}^{N-1}\left(\nabla U^{k+1}, \nabla \mathcal{I}_{h}\left(W_{+}^{\prime}\left(U^{k+1}\right)+W_{-}^{\prime}\left(U^{k}\right)\right)\right) \\
& =\mathcal{I}_{1}-\mathcal{I}_{2} .
\end{aligned}
$$

\footnotetext{
${ }^{1}$ In the next section, it will become evident that already the energy estimate implies strict positivity of discrete solutions if (H1) holds. Hence, this assumption does not mean any restriction.
} 
It remains to estimate $\mathcal{I}_{2}$. Since the Euclidean scalar product $\nabla U \cdot \nabla V$ remains invariant under orthogonal transformations on each element $E_{k}$, we observe that

$$
\begin{aligned}
\mathcal{I}_{2}= & \tau \sum_{k=0}^{N-1}\left\langle\hat{\nabla} U^{k+1}, \hat{\nabla} \mathcal{I}_{h}\left(W_{+}^{\prime}\left(U^{k+1}\right)+W_{-}^{\prime}\left(U^{k}\right)\right)\right\rangle \\
= & \tau \sum_{k=0}^{N-1}\left\langle\hat{\nabla} U^{k+1}, \operatorname{diag}\left(\partial W_{+}^{\prime}\left(U^{k+1}\right)\right) \hat{\nabla} U^{k+1}\right\rangle \\
+ & \tau \sum_{k=0}^{N-1}\left\langle\hat{\nabla} U^{k+1}, \operatorname{diag}\left(\partial W_{-}^{\prime}\left(U^{k}\right)\right) \hat{\nabla} U^{k}\right\rangle \\
= & \tau \sum_{k=1}^{N-1}\left\{\left\langle\hat{\nabla} U^{k}, \operatorname{diag}\left(\partial\left(W_{+}^{\prime}\left(U^{k}\right)\right)\right) \hat{\nabla} U^{k}\right\rangle\right. \\
& \left.+\left\langle\hat{\nabla} U^{k+1}, \operatorname{diag}\left(\partial W_{-}^{\prime}\left(U^{k}\right)\right) \hat{\nabla} U^{k}\right\rangle\right\} \\
+ & \tau\left\langle\hat{\nabla} U^{N}, \operatorname{diag}\left(\partial W_{+}^{\prime}\left(U^{N}\right)\right) \hat{\nabla} U^{N}\right\rangle \\
+ & \tau\left\langle\hat{\nabla} U^{1}, \operatorname{diag}\left(\partial W_{-}^{\prime}\left(U^{0}\right)\right) \hat{\nabla} U^{0}\right\rangle \\
= & \mathcal{I}_{2}^{1}+\mathcal{I}_{2}^{2}+\mathcal{I}_{2}^{3} .
\end{aligned}
$$

Let us distinguish two cases. First, we assume that hypothesis (H1) holds. We may estimate term $\mathcal{I}_{2}^{1}$ as follows:

$$
\begin{aligned}
\mathcal{I}_{2}^{1} \geq \tau \sum_{k=1}^{N-1}\{ & \left\langle\hat{\nabla} U^{k}, \operatorname{diag}\left(\partial W_{+}^{\prime}\left(U^{k}\right)\right) \hat{\nabla} U^{k}\right\rangle \\
& \left.-\frac{\varepsilon}{2}\left\langle\hat{\nabla} U^{k}, \operatorname{diag}^{2}\left(\partial W_{-}^{\prime}\left(U^{k}\right)\right) \hat{\nabla} U^{k}\right\rangle-\frac{1}{2 \varepsilon}\left\|\nabla U^{k+1}\right\|_{L^{2}(\Omega)}^{2}\right\} .
\end{aligned}
$$

Now we use the estimate on difference quotients:

$$
\left|\partial_{i} W_{-}^{\prime}(\cdot)\right|^{2} \leq C\left(\varrho_{1}, \varrho_{2}\right) \frac{H_{2}^{2}}{H_{1}}\left(\partial_{i} W_{+}^{\prime}(\cdot)+H_{1} \cdot \varrho_{1}\right),
$$

which holds for positive arguments provided $\varrho_{1}>2 \varrho_{2}+1$ as will be proven in the next lemma.

Choosing $\varepsilon$ in an appropriate way and collecting the remaining terms, case 1 can be settled.

If hypothesis (H2) holds, $\mathcal{I}_{2}^{1}$ can be estimated by Young's inequality in a standard way:

$$
\mathcal{I}_{2}^{1} \geq \tau \sum_{k=1}^{N-1}\left\langle\hat{\nabla} U^{k}, \operatorname{diag}\left(\partial W_{+}^{\prime}\left(U^{k}\right)\right) \hat{\nabla} U^{k}\right\rangle-K_{2} \tau \sum_{K=1}^{N}\left\|\nabla U^{k}\right\|^{2} .
$$

$\mathcal{I}_{2}^{2}$ is a nonnegative term, and $\mathcal{I}_{2}^{3}$ can be estimated by Young's inequality. This gives the assertion.

For completeness, we state another lemma. 
Lemma 4.4. Assume that hypothesis (H1) holds. Then there exists a positive constant $C=C\left(\varrho_{1}, \varrho_{2}\right)$ such that for positive numbers $u_{1}<u_{2}$ we have

$$
\left(\frac{W_{-}^{\prime}\left(u_{2}\right)-W_{-}^{\prime}\left(u_{1}\right)}{u_{2}-u_{1}}\right)^{2} \leq C \frac{H_{2}^{2}}{H_{1}}\left(\frac{W_{+}^{\prime}\left(u_{2}\right)-W_{+}^{\prime}\left(u_{1}\right)}{u_{2}-u_{1}}+H_{1} \varrho_{1}\right) .
$$

Proof. Lemma 4.2 in [19] implies the existence of a positive constant $\hat{C}\left(\varrho_{1}, \varrho_{2}\right)$ such that

$$
\left(\frac{W_{-}^{\prime}\left(u_{2}\right)-W_{-}^{\prime}\left(u_{1}\right)}{u_{2}-u_{1}}\right)^{2} \leq C \frac{H_{2}^{2}}{H_{1}} u_{1}^{\varrho_{1}-1-2 \varrho_{2}} \frac{W_{+}^{\prime}\left(u_{2}\right)-W_{+}^{\prime}\left(u_{1}\right)}{u_{2}-u_{1}} .
$$

Due to (H1), $u_{1}^{\varrho_{1}-1-2 \varrho_{2}}<1$ for $u_{1} \leq 1$. For $u_{1}>1$, a number $\xi \in\left(u_{1}, u_{2}\right)$ exists such that

$$
\frac{W_{+}^{\prime}\left(u_{2}\right)-W_{+}^{\prime}\left(u_{1}\right)}{u_{2}-u_{1}}=W_{+}^{\prime \prime}(\xi)=H_{1} \cdot \varrho_{1} \xi^{-\varrho_{1}-1} .
$$

Hence, for $u_{1}>1$ we have

$$
\left(\frac{W_{-}^{\prime}\left(u_{2}\right)-W_{-}^{\prime}\left(u_{1}\right)}{u_{2}-u_{1}}\right)^{2} \leq C\left(\varrho_{1}, \varrho_{2}\right) H_{2}^{2} \cdot \varrho_{1} \cdot \xi^{-2-2 \varrho_{2}} \leq C\left(\varrho_{1}, \varrho_{2}\right) \cdot H_{2}^{2} \cdot \varrho_{1} .
$$

This gives the result.

\section{Estimates on NONNEGATIVITY OR POSITIVITY OF DISCRETE SOLUTIONS}

Besides slight modifications, the approach used in [19] to prove nonnegativity or strict positivity of discrete solutions in the one-dimensional setting carries over to the multi-dimensional case. For the reader's convenience, we gather here the main results, without giving proofs.

If hypothesis (H1) holds, the energy estimate implies strict positivity of discrete solutions independently of the dimension, provided the initial data are strictly positive. If hypothesis (H2) holds, results on nonnegativity or positivity can be obtained by use of the entropy estimate. The following theorem holds.

Theorem 5.1 (Existence of nonnegative discrete solutions $U_{\tau h}^{\sigma}$ ). Let $\mathcal{T}_{h}$ be an admissible triangulation of $\Omega$ satisfying (T1), and assume (M1) and (H2) hold. For arbitrary $\varepsilon>0$, there exists a positive control parameter $\sigma_{0}$, which only depends on $d, n, \varepsilon, h, T, K_{2}$ and initial data $u_{0} \geq 0$, such that:

For every $0<\sigma<\sigma_{0}$ discrete entropy-mobility pairs $\left(G_{\sigma}, M_{\sigma}\right)$ can be constructed having the property that on $\Omega_{T}$ the corresponding discrete solutions $U_{\tau h}^{\sigma}$ of equation (5) -(6) satisfy

- $U_{\tau h}^{\sigma}>-\varepsilon$ if $u^{0} \geq 0$ and $0<n<2$,

- $U_{\tau h}^{\sigma}>-\varepsilon$ if $u^{0} \geq \sigma_{0}$ and $n=2$, and

- $U_{\tau h}^{\sigma}>\sigma / 2$ if $u^{0} \geq \sigma_{0}$ and $n>2$.

Remarks. 1. As the proof of Theorem 5.1 is nearly identical to the corresponding result in the case $W \equiv 0$, which can be found in [18], we refer to that paper for the details.

2. Let us emphasize that the control parameter $\sigma_{0}$ does not depend on the timeincrement $\tau$. In order to ensure a lower bound $U_{\tau h}^{\sigma} \geq-\varepsilon, \sigma_{0}$ has to tend to zero as $h$ tends to zero.

So let us assume now that hypothesis (H1) holds. We have 
Theorem 5.2 (Existence of strictly positive discrete solutions). Let $\mathcal{T}_{h}$ be an admissible triangulation of $\Omega$ satisfying (T1), and assume that (M1) and (H1) hold, that initial data are strictly positive and that $\left\|U_{h}^{0}\right\|_{1}^{2}+\int_{\Omega} \mathcal{I}_{h} W\left(U_{h}^{0}\right)$ is uniformly bounded for $h \rightarrow 0$. Then for arbitrary $h, \tau, T$, a solution $U_{h}$ to the discrete system (5) -(6) exists globally on $\Omega_{T}$. Moreover, for arbitrary $h$ there is a positive number $\gamma(h)$ such that $U_{h} \geq \gamma(h)$ on $\Omega_{T}$.

Remarks. 1. This result can be proven along the lines of Theorem 5.2 in [19]. It is worth noting one difference between the case of one and multiple space dimensions: As the energy estimate does not provide more than $L^{\infty}\left(I ; H^{1,2}(\Omega)\right)$-regularity, it is no longer possible to prove the existence of a positive lower bound on discrete solutions uniformly for $h \rightarrow 0$.

2. Observe that we may obtain a result in the spirit of Theorem 5.2 also in the case that the discrete mobility $M(\cdot)$ is not necessarily part of an admissible entropymobility pair. However, in that case we will lose those integrability properties of the discrete Laplacian of discrete solutions which are essential to pass to the limit as $h \rightarrow 0$. Therefore, we always assume $M(\cdot)$ to be given as part of an admissible entropy-mobility pair.

\section{Higher Regularity of Discrete SOlutions UNifORMLY AS $h \rightarrow 0$}

In this section, we will prove the key results on regularity of discrete solutions which will enable us in the sequel to pass to the limit as $h \rightarrow 0$ in the weak formulation (5)-(6).

The following theorem holds:

Theorem 6.1. Let $d \in\{2,3\}$ and let $\left(U_{h}\right)_{h \rightarrow 0}$ be a sequence of discrete solutions to the system (5) - (6) which satisfy both the energy and the entropy estimate. Then

$$
\left(U_{h}\right)_{h \rightarrow 0} \text { is uniformly bounded in } L^{2}\left(I ; W^{1, p}(\Omega)\right)
$$

for arbitrary $p< \begin{cases}\infty & \text { if } d=2, \\ \frac{2 d}{d-2} & \text { if } d=3 .\end{cases}$

By Sobolev's imbedding result (recall that mass is conserved!), we immediately obtain

Corollary 6.2. Let $d \in\{2,3\}$ and let $\left(U_{h}\right)_{h \rightarrow 0}$ be a sequence of discrete solutions satisfying both the energy and the entropy estimate. Then

$$
\left(U_{h}\right)_{h \rightarrow 0} \text { is uniformly bounded in } L^{2}\left(I ; C^{\beta}(\Omega)\right)
$$

for arbitrary $\beta<2-\frac{d}{2}$.

Proof of Theorem [6.1. Combining the entropy estimate (20), the energy estimate (18), and a discrete version of Gronwall's lemma, we observe that the discrete Laplacian

$$
-\Delta_{h} U_{h}:=P_{h}-\mathcal{I}_{h}\left(W_{+}^{\prime}\left(U_{h}\right)+W_{-}^{\prime}\left(U_{h}(\cdot-\tau)\right)\right)
$$

is uniformly bounded in $L^{2}\left(I ; L^{2}(\Omega)\right)$ for $h$ tending to zero. Hence, it will be sufficient to prove, for

$$
p< \begin{cases}\infty & \text { if } d=2, \\ \frac{2 d}{d-2} & \text { if } d=3\end{cases}
$$


the existence of a positive constant $C$, depending only on the data, such that

$$
\|\nabla U\|_{L^{p}(\Omega)} \leq C \cdot\|F\|_{L^{2}(\Omega)}
$$

for arbitrary $(F, U) \in V^{h} \times V^{h}$, which satisfy

$$
\left(\nabla U, \nabla \Psi_{h}\right)=\left(F, \Psi_{h}\right)_{h} \quad \text { and } \quad \int_{\Omega} U=0
$$

for arbitrary $\Psi_{h} \in V^{h}$.

To this purpose, consider functions $\hat{U} \in V^{h}$ and $u \in H^{1}(\Omega)$ which have mean value zero on $\Omega$ and which solve the auxiliary problems

$$
\left(\nabla \hat{U}, \nabla \Psi_{h}\right)=\left(F, \Psi_{h}\right) \quad \forall \Psi_{h} \in V^{h}
$$

and

$$
(\nabla u, \nabla \Psi)=(F, \Psi) \quad \forall \Psi \in H^{1,2}(\Omega),
$$

respectively. Estimate (3) on the lumped masses scalar product implies

$$
\left|\left(\nabla U-\nabla \hat{U}, \nabla \Psi_{h}\right)\right| \leq C \cdot h\left\|\Psi_{h}\right\|_{H^{1,2}(\Omega)} \cdot\|F\|_{L^{2}(\Omega)} \quad \forall \Psi_{h} \in V^{h} .
$$

The choice $\Psi_{h}=U-\hat{U}$ yields

$$
\|\nabla U-\nabla \hat{U}\|_{L^{2}(\Omega)} \leq C \cdot h \cdot\|F\|_{L^{2}(\Omega)} .
$$

Elliptic regularity theory $\mathrm{l}^{\mathrm{B}}$ and standard estimates on the convergence of linear finite elements (cf. [11]) imply

$$
\|\nabla \hat{U}-\nabla u\|_{L^{2}(\Omega)} \leq C \cdot h \cdot\|F\|_{L^{2}(\Omega)} .
$$

Hence

$$
\|\nabla U-\nabla u\|_{L^{2}(\Omega)} \leq C \cdot h \cdot\|F\|_{L^{2}(\Omega)} .
$$

Interpolation results for linear finite elements (cf. also the subsequent Lemma 6.3) combined with (36) lead to the estimate

$$
\left\|\nabla\left(\mathcal{I}_{h} u\right)-\nabla U\right\|_{L^{2}(\Omega)} \leq C \cdot h \cdot\|F\|_{L^{2}(\Omega)} .
$$

From this, we infer the elementwise estimate

$$
\left|\nabla\left(\mathcal{I}_{h} u\right)-\nabla U\right|_{E} \leq C \cdot h^{\frac{2-d}{2}} \cdot\|F\|_{L^{2}(\Omega)} .
$$

Indeed, $\nabla\left(\mathcal{I}_{h} u\right)-\nabla U$ is constant on each element, and from (37) we obtain

$$
h^{d}\left|\nabla\left(\mathcal{I}_{h} u\right)-\nabla U\right|_{E}^{2} \leq C \cdot h^{2}\|F\|_{L^{2}(\Omega)}^{2} .
$$

This implies (38). Hence, for $V:=\mathcal{I}_{h} u-U \in V^{h}$, we obtain in space dimension $d=2$,

$$
\|\nabla V\|_{L^{\infty}(\Omega)} \leq C \cdot\|F\|_{L^{2}(\Omega)}
$$

In space dimension $d=3$, we proceed as follows:

$$
\begin{aligned}
\int_{\Omega}|\nabla V|^{\frac{2 d}{d-2}} d x & \leq \sup _{\Omega}|\nabla V|^{\frac{2 d}{d-2}-2} \int_{\Omega}|\nabla V|^{2} \\
& \leq C \cdot\|F\|_{L^{2}(\Omega)}^{\frac{2 d}{d-2}},
\end{aligned}
$$

\footnotetext{
${ }^{2}$ Note that here we are implicitly using the assumption that the domain $\Omega$ is convex.
} 
where we used (38). Hence

$$
\|\nabla V\|_{L^{\frac{2 d}{d-2}}} \leq C \cdot\|F\|_{L^{2}(\Omega)}
$$

Now, writing

$$
\|\nabla U\|_{L^{p}(\Omega)} \leq\|\nabla V\|_{L^{p}(\Omega)}+\left\|\nabla\left(\mathcal{I}_{h} u-u\right)\right\|_{L^{p}(\Omega)}+\|\nabla u\|_{L^{p}(\Omega)}
$$

for $p<\frac{2 d}{d-2}$, we get the result provided $\left\|\nabla\left(\mathcal{I}_{h} u-u\right)\right\|_{L^{p}(\Omega)}$ can be bounded accordingly. By the subsequent Lemma 6.3 we have, for each $p<\frac{2 d}{d-2}$,

$$
\left\|\nabla\left(\mathcal{I}_{h} u-u\right)\right\|_{L^{p}(\Omega)} \leq C \cdot h^{\varepsilon(p)}\|u\|_{H^{2}(\Omega)} \leq C \cdot h^{\varepsilon(p)}\|F\|_{L^{2}(\Omega)}
$$

where $\varepsilon(p)$ is a small positive number. This proves the theorem.

For the sake of completeness, let us state the following interpolation result

Lemma 6.3. Let $\mathcal{T}_{h}$ be a regular triangulation of the domain $\Omega$ with simplicial elements. Let $\mathcal{I}_{h}$ be the corresponding nodal interpolation operator. Then, for $v \in H^{2}(\Omega)$ and

$$
2 \leq q \begin{cases}<\infty & \text { if } d=2 \\ <6 & \text { if } d=3\end{cases}
$$

the following error estimate holds:

$$
\left\|\nabla \mathcal{I}_{h} v-\nabla v\right\|_{L^{q}(\Omega)} \leq C \cdot h^{\frac{\varepsilon(q)}{2}}\|v\|_{H^{2}(\Omega)} .
$$

Here, the nonnegative exponent $\varepsilon(q)$ is given by

$$
\varepsilon(q)=\frac{2 d-q(d-2)}{q} .
$$

Proof. We use the identity $q=\frac{2 d}{d-2+\varepsilon(q)}$, and we deduce from Theorem 3.1.5 in [11] the existence of a positive constant $C$ such that

$$
\left\|\nabla \mathcal{I}_{h} v-\nabla v\right\|_{L^{q}(E)} \leq C \cdot h^{\frac{\varepsilon(q)}{2}}\|v\|_{H^{2}(E)} .
$$

Summing up over all elements yields

$$
\left\|\nabla \mathcal{I}_{h} v-\nabla v\right\|_{L^{q}(\Omega)}^{q} \leq C \cdot h^{\frac{\varepsilon(q) q}{2}} \sum_{E \in \mathcal{T}_{h}}\|v\|_{H^{2}(E)}^{q} .
$$

Since $q \geq 2$, we may apply the inequality

$$
\left(\sum_{i=1}^{k}\left|a_{i}\right|^{q}\right)^{\frac{1}{q}} \leq\left(\sum_{i=1}^{k}\left|a_{i}\right|^{2}\right)^{\frac{1}{2}}
$$

which holds for arbitrary $k \in \mathbb{N}$. Hence, (43) follows. 


\section{BASiC A PRIORI ESTIMATES - COMPACTNESS IN TIME}

Before formulating a result on compactness in time, let us state a few preliminary observations. Lemma 3.1 implies

(A1) $\exists C>0$, such that for all $U \in V^{h}$

$$
\int_{\Omega} M_{\sigma}(U) d x \leq C\left(\int_{\Omega}|U|^{n} d x+1\right)
$$

if $M_{\sigma}(U), 0<\sigma<1$, is the entropy consistent field of mobility matrices corresponding to $\mathcal{M}(u)=(u)_{+}^{n}, n \in \mathbb{R}^{+}$.

If discrete initial data satisfy $\int_{\Omega}\left\{\left|\nabla U_{h}^{0}\right|^{2}+\mathcal{I}_{h} W\left(U_{h}^{0}\right)+\mathcal{I}_{h} G\left(U_{h}^{0}\right)\right\} \leq C<\infty$ uniformly for $h \rightarrow 0$, then the following regularity property follows as a consequence of Theorem 6.1. Corollary 6.2 and the energy estimate.

(A2) The discrete solutions $\left(U_{h}\right)_{h \rightarrow 0}$ are uniformly bounded in $L^{2}\left(I ; C^{\beta}(\Omega)\right) \cap L^{2}\left(I ; W^{1, p}(\Omega)\right) \cap L^{\infty}\left(I ; H^{1,2}(\Omega)\right)$ for $\beta<$ $2-\frac{d}{2}$ and $p< \begin{cases}\infty & \text { if } d=2, \\ \frac{2 d}{d-2} & \text { if } d=3 .\end{cases}$

From (A1), (A2), and Corollary 4.2 we infer by straightforward calculations for discrete solutions $\left(U_{h}, P_{h}\right)_{h \rightarrow 0}$ in the limit as $h \rightarrow 0$ that

$$
\left|M_{\sigma}\left(U_{h}\right) \nabla P_{h}\right| \text { is uniformly bounded in } L^{2}\left(I ; L^{q}(\Omega)\right)
$$

for each $q<\frac{4 d}{2 d+n(d-2)}$ if $d=2$ and $n>0$, or if $d=3$ and $0<n<4$.

The following estimate on compactness in time can be established:

Lemma 7.1 (Compactness in time). Let $\left(U_{h}, P_{h}\right)_{h \rightarrow 0}$ be a sequence of discrete solutions satisfying (A2). Let $s<T$ be a positive number, and assume (A1) holds.

If $d=2$ and $n>0$ or if $d=3$ and $0<n<4$, a positive constant $C=C\left(T, u_{0}\right)$ exists such that

$$
\int_{0}^{T-s}\left\|U_{h}(t+s, x)-U_{h}(t, x)\right\|_{h}^{2} d t \leq C \cdot s .
$$

Proof. Let us first prove the result for values $s=l \tau, l \leq N$ a positive integer. We will abbreviate $U_{h}$ by $U$. For a fixed number $j$ satisfying $0 \leq j \leq N-l$ we choose

$$
\Theta:=U^{j+l}-U^{j}
$$

in equation (5), multiply by $\tau$ and sum over $k=j-1, \cdots, j+l-1$. This implies

$$
\begin{aligned}
& \tau \sum_{k=j-1}^{j+l-1}\left(\frac{U^{k+1}-U^{k}}{\tau}, U^{j+l}-U^{j}\right)_{h} \\
& \quad=-\tau \sum_{k=j-1}^{j+l-1} \int_{\Omega} M\left(U^{k+1}\right) \nabla P^{k+1} \nabla\left(U^{j+l}-U^{j}\right) .
\end{aligned}
$$


As the term on the left-hand side is equal to $\left(U^{j+l}-U^{j}, U^{j+l}-U^{j}\right)_{h}$, it follows that

$$
\begin{aligned}
& \left(U^{j+l}-U^{j}, U^{j+l}-U^{j}\right)_{h} \\
& \quad \leq \tau \sum_{k=0}^{l}\left(\int_{\Omega}\left|M\left(U^{j+k}\right) \nabla P^{j+k}\right|^{q}\right)^{\frac{1}{q}}\left(\int_{\Omega}\left|\nabla\left(U^{j+l}-U^{j}\right)\right|^{q^{\prime}}\right)^{\frac{1}{q^{\prime}}}
\end{aligned}
$$

for arbitrary $q<\frac{4 d}{2 d-n(d-2)}$. Summing from $j=1$ to $N-l$ gives

$$
\begin{aligned}
& \tau \sum_{j=1}^{N-l}\left(U^{j+l}-U^{j}, U^{j+l}-U^{j}\right)_{h} \\
& \leq C \cdot \tau \sum_{k=1}^{l}\left(\tau \sum_{j=1}^{N-l}\left(\int_{\Omega}\left|M\left(U^{j+k}\right) \nabla P^{j+k}\right|^{q}\right)^{\frac{2}{q}}\right)^{\frac{1}{2}} \\
& \quad \times\left(\tau \sum_{j=1}^{N-l}\left(\int_{\Omega}\left|\nabla\left(U^{j+l}-U^{j}\right)\right|^{q^{\prime}}\right)^{\frac{2}{q^{\prime}}}\right)^{\frac{1}{2}} \\
& \leq C \cdot(l+1) \tau,
\end{aligned}
$$

which is inequality (46) for $s=l \tau$. Since the remainder of the proof is similar to that of Lemma 3.3 in [18], we omit it here.

Remark. Observe that $q^{\prime}=\frac{4 d}{2 d-n(d-2)}<\frac{2 d}{d-2}$ if $n<\frac{4}{d-2}$. This explains the restriction that $n<4$ if $d=3$.

\section{A CONVERGEnCE AND EXIstence RESUlt}

In this section, we will present the main result of this paper. First, we need some notation. We will consider a sequence of discrete solutions $\left(U_{h}, P_{h}\right)_{h \rightarrow 0}$ which converges to a pair of functions $(u, p)$ in a sense to be specified later on. To avoid clumsy notation, we assume implicitly that the functions $U_{h}$ are bounded from below by — not necessarily optimal - lower bounds $\kappa(h)$ which converge monotonically to zero with $h$. The existence of those lower bounds follows by the results on discrete nonnegativity in section 5. Moreover, we write $\tau$ for the time-increment $\tau(h)$ and $\sigma$ for the regularization parameter $\sigma(h)$ occurring in the definition of admissible entropy-mobility pairs. We will use the following abbreviations:

Definition 8.1. For $\delta>0$, we consider the following sets:

$$
\begin{aligned}
S_{\delta} & :=\left\{(t, x) \in \Omega_{T} \mid u(t, x) \geq \delta\right\}, \\
S_{\delta}(t) & :=\{x \in \Omega \mid u(t, x) \geq \delta\}, \\
S_{\delta}^{\mathcal{T}_{h}} & :=\left\{(t, x) \in \Omega_{T} \mid \exists E \in \mathcal{T}_{h} \text { such that } x \in E \text { and }\left.U_{h}(t, \cdot)\right|_{E} \geq \delta\right\}, \\
S_{\delta}^{\mathcal{T}_{h}}(t) & :=\left\{x \in \Omega \mid \exists E \in \mathcal{T}_{h} \text { such that } x \in E \text { and }\left.U_{h}(t, \cdot)\right|_{E} \geq \delta\right\} .
\end{aligned}
$$

By $\mu_{d}(A)$, we denote the $d$-dimensional Lebesgue-measure of the set $A$.

We have

Theorem 8.2. Let $\Omega$ be a convex polygonal domain in $\mathbb{R}^{d}, d \in\{2,3\}$, and let $u_{0} \in H^{1}\left(\Omega ; \mathbb{R}_{0}^{+}\right) \cap C(\Omega)$ be given. Suppose $n \in \mathbb{R}^{+}$if $d=2$ or $n \in(0,4)$ if $d=3$. 
Assume that (H1) or (H2) is satisfied and that a positive constant $C$ exists such that discrete initial data $U_{h}^{0}:=\mathcal{I}_{h} u_{0}$ satisfy

$$
\int_{\Omega}\left\{\left|\nabla U_{h}^{0}\right|^{2}+\mathcal{I}_{h} W\left(U_{h}^{0}\right)+\mathcal{I}_{h} G\left(U_{h}^{0}\right)\right\} \leq C<\infty
$$

uniformly for $h \rightarrow 0$. Assume that $\mathcal{T}_{h}$ satisfies (T1) and that $\left(U_{h}, P_{h}\right)_{h \rightarrow 0}$ is a sequence of discrete solutions to equations (5) and (6). Then, there exist a set $\mathcal{S} \subset I, \mu_{1}(\mathcal{S})=\mu_{1}(I)$, a nonnegative function $u \in L^{\infty}\left(I ; H^{1,2}(\Omega)\right) \cap L^{2}\left(I ; H^{2}(\Omega)\right)$ and a function $p:[u>0] \rightarrow \mathbb{R}$ with the following properties: For $t \in \mathcal{S}$, the function $u(t, \cdot)$ is Hölder continuous on $\Omega$ with exponent $0<\beta<2-\frac{d}{2}$, the function $p(t, \cdot)$ is an element of $H_{\mathrm{loc}}^{1}([u(t, \cdot)>0])$, and $(u, p)$ solves (2) in the following sense:

$$
\begin{gathered}
\int_{\Omega_{T}}\left(u-u_{0}\right) \frac{\partial}{\partial t} \psi d x d t=\int_{[u>0]} \mathcal{M}(u) \cdot \nabla p \nabla \psi d x d t \\
\text { for all } \psi \in C^{1}\left((0, T) ; H^{1,2}(\Omega)\right) \text { satisfying } \psi(T)=0, \\
p(t, \cdot)=-\Delta u(t, \cdot)+W^{\prime}(u(t, \cdot)) \\
\text { on }[u(t, \cdot)>0] \text { for } t \in \mathcal{S} .
\end{gathered}
$$

Moreover, for a subsequence $h \rightarrow 0$ the following convergence results hold true:

- $U_{h} \rightarrow u$ strongly $L^{2}\left(I ; C^{\beta}(\Omega)\right)$ for any $0<\beta<2-\frac{d}{2}$,

- $P_{h}-\mathcal{I}_{h} W_{+}^{\prime}\left(U_{h}\right)-\mathcal{I}_{h} W_{-}^{\prime}\left(U_{h}(\cdot-\tau, \cdot)\right) \rightarrow-\Delta u$ weakly in $L^{2}\left((\varepsilon, T) ; L^{2}(\Omega)\right)$ for arbitrary $0<\varepsilon<T$,

- $\mathcal{I}_{h} W_{+}^{\prime}\left(U_{h}\right) \chi_{\left[S_{\delta} \cap S_{\frac{\delta}{\tau}}^{T_{h}}\right]} \rightarrow W_{+}^{\prime}(u) \chi_{\left[S_{\delta}\right]}$ strongly in $L^{2}\left(\Omega_{T}\right)$,

- $\mathcal{I}_{h} W_{-}^{\prime}\left(U_{h}(\cdot-\tau, \cdot)\right) \chi_{\left[S_{\frac{\delta}{4}}^{\mathcal{T}_{h}}\right]}(\cdot-\tau, \cdot) \chi_{\left[S_{\delta}\right]}(\cdot, \cdot) \rightarrow W_{-}^{\prime}(u) \chi_{\left[S_{\delta}\right]}$ strongly in the space $L^{2}\left((\varepsilon, T) ; L^{2}(\Omega)\right)$ for arbitrary $0<\varepsilon<T$,

- $P_{h}(t, \cdot) \rightarrow p$ weakly in $H^{1}\left(S_{\delta}(t)\right)$ for arbitrary $\delta>0$ and $t \in \mathcal{S}$.

Remarks. 1. In the continuous setting, Theorem 8.2 extends previous existence and nonnegativity results (cf. [10]) to the parameter range $n \in(0,1 / 8]$. In addition, it is the first existence result for equation (11) with a pressure function $p$ containing singular lower order terms.

2. For fourth order degenerate parabolic equations like (1), uniqueness results are only known within the class of strictly positive solutions (cf. [8] and [19]). Therefore, the aforementioned convergence result only holds for subsequences. Nevertheless, it is conjectured that solutions in the continuous setting satisfying an entropy estimate are unique.

The proof of this theorem relies on a number of auxiliary results which will be listed and proven below.

The first result is about preliminary convergence results for discrete solutions $\left(U_{h}\right)_{h \rightarrow 0}$ and discrete fluxes $\left(J_{h}\right)_{h \rightarrow 0}$. It can be obtained along the lines of the proof for Theorem 8.1 in [18] using in addition Lemma 7.1, Corollary 6.2] and the compactness of the imbedding $C^{\alpha}(\Omega) \hookrightarrow C^{\beta}(\Omega)$ for $\alpha>\beta$.

Lemma 8.3. Under the assumptions of Theorem 8.2, functions

$$
u \in L^{\infty}\left(I ; H^{1,2}(\Omega)\right) \cap L^{2}\left(I ; H^{2}(\Omega)\right) \text { and } J \in L^{2}\left(I ; L^{q_{1}}(\Omega)^{d}\right),
$$

$q_{1}<\frac{4 d}{2 d+(d-2) n}$ exist such that for a subsequence of discrete solutions $\left(U_{h}, P_{h}\right)$ we have, in the limit $h \rightarrow 0$, 
i) $U_{h} \rightarrow u$ strongly in $L^{2}\left(I ; C^{\beta}(\Omega)\right)$ for $0<\beta<2-\frac{d}{2}$,

ii) $P_{h}-\mathcal{I}_{h} W_{+}^{\prime}\left(U_{h}\right)-\mathcal{I}_{h} W_{-}^{\prime}\left(U_{h}(\cdot-\tau, \cdot)\right) \rightarrow-\Delta u$ weakly in $L^{2}\left((\varepsilon, T) ; L^{2}(\Omega)\right)$ for arbitrary $0<\varepsilon<T$, and

iii) $J_{h}:=M_{\sigma}\left(U_{h}\right) \cdot \nabla P_{h} \rightarrow J$ weakly in $L^{2}\left(I ; L^{q_{1}}(\Omega)\right)$ for $q_{1}<\frac{4 d}{2 d+(d-2) n}$.

Furthermore, $u$ is nonnegative, and $u_{t}=\operatorname{div} J$ in the following weak sense:

$$
\begin{gathered}
\qquad \int_{\Omega_{T}}\left(u-u_{0}\right) \frac{\partial}{\partial t} \psi d x d t=\int_{[u>0]} J \nabla \psi d x d t \\
\text { for all } \psi \in C^{1}\left((0, T) ; H^{1,2}(\Omega)\right) \text { satisfying } \psi(T)=0 .
\end{gathered}
$$

A combination of the Fréchet-Kolmogorov theorem (cf. 22]) with the strong $L^{2}\left(I ; C^{\beta}(\Omega)\right)$-convergence of $\left(U_{h}\right)_{h \rightarrow 0}$ implies the following corollary.

Corollary 8.4. Under the assumptions of Lemma 8.3, there is a subsequence $h \rightarrow 0$ such that $\left(U_{h}(t-\tau(h), \cdot)\right)_{h \rightarrow 0}$ uniformly converges to $u(t, \cdot)$ in $C^{\beta}(\Omega)$ for all $0<$ $\beta<2-\frac{d}{2}$ and for almost every $t \in I$.

The next ingredient is a convergence result for the field of discrete mobility matrices $M_{\sigma}\left(U_{h}\right)$. By a slight misuse of notation, we identify the field of matrices $M_{\sigma}\left(U_{h}\right) \in \bigotimes_{k=1}^{\left|\mathcal{T}_{h}\right|} \mathbb{R}^{d \times d}$ with a matrix valued function $\bar{M}_{\sigma}\left(U_{h}\right): \Omega \rightarrow \mathbb{R}_{\text {sym }}^{d \times d}$ which is elementwise constant. The lemma reads as follows:

Lemma 8.5. Let $\mathcal{T}_{h}$ be a regular triangulation of $\Omega$ satisfying (T1), and let $\left(U_{h}\right)_{h \rightarrow 0}$ be a sequence of discrete solutions which strongly converges to $u \in L^{2}\left(I ; C^{\beta}(\Omega)\right)$, $\beta \in\left(0,2-\frac{d}{2}\right)$, as $h \rightarrow 0$. Assume further that $\left(U_{h}\right)_{h \rightarrow 0}$ is uniformly bounded in $L^{\infty}\left(I ; H^{1,2}(\Omega)\right)$. If $\mathcal{M}(s)=(s)_{+}^{n}$ with $n$ as in Theorem 8.2 then the field of discrete mobility matrices $M_{\sigma}\left(U_{h}\right)$ as defined in section 3 strongly converges to $\mathcal{M}(u) \cdot \mathrm{Id}$ in $L^{p}\left(\Omega_{T}\right)$ for any $p< \begin{cases}\infty & \text { if } d=2, \\ \frac{6}{n} & \text { if } d=3 .\end{cases}$

Proof. Let us prove the result first for $p=1$. We have to estimate the quantity

$$
\int_{\Omega_{T}}\left|M_{\sigma}\left(U_{h}\right)-\mathcal{M}(u) \cdot \mathrm{Id}\right|=\sum_{k=1}^{N(h)} \sum_{i=1}^{\left|\mathcal{T}_{h}\right|} \int_{I_{k}} \int_{E_{i}}\left|M_{\sigma}\left(U_{h}\right)-\mathcal{M}(u) \cdot \mathrm{Id}\right| .
$$

Here $I_{k}, k=1, \cdots, N(h)$, denote the subintervals of $I$ on which $U_{h}$ is constant, and $E_{i}, i=1, \cdots,\left|\mathcal{T}_{h}\right|$, stand for the elements of the triangulation $\mathcal{T}_{h}$. By construction, $M_{\sigma}\left(U_{h}\right)$ is a constant symmetric positive semi-definite matrix on $E_{k i}(h):=I_{k} \times$ $E_{i} \subset \Omega_{T}$. It will be sufficient to estimate on each element the difference between $\mathcal{M}(u)$ and the eigenvalues of this matrix. Since the latter are given by $\varrho_{\sigma}\left(U_{j}, U_{0}\right)$, $j=1, \cdots, d$ (cf. section [3), we may assume without loss of generality that the elements $E_{i}$ are rectangular and that their vertices $x_{0}\left(E_{i}\right)=0, \cdots, x_{d}\left(E_{i}\right)$ lie on the coordinate axes. Using the mean-value theorem, we obtain for the $j^{\text {th }}$ component, $j=1, \cdots, d$,

$$
\int_{E_{k i}(h)}\left|M_{\sigma j j}\left(U_{h}\right)-\mathcal{M}(u)\right|=\int_{E_{k i}(h)}\left|m_{\sigma}\left(\xi_{j}^{k i}\right)-\mathcal{M}(u)\right|
$$


with $\xi_{j}^{k i} \in\left[U_{h}\left(k \tau, x_{0}\left(E_{i}\right)\right), U_{h}\left(k \tau, x_{j}\left(E_{i}\right)\right)\right]$. Hence

$$
\begin{aligned}
\int_{E_{k i}(h)} & \left|M_{\sigma j j}\left(U_{h}\right)-\mathcal{M}(u)\right| \\
\leq & \int_{E_{k i}(h)}\left|\mathcal{M}(u)-\mathcal{M}\left(\xi_{j}^{k i}\right)\right|+\int_{E_{k i}(h)}\left|\mathcal{M}\left(\xi_{j}^{k i}\right)-m_{\sigma}\left(\xi_{j}^{k i}\right)\right| \\
= & \mathcal{I}_{1}+\mathcal{I}_{2} .
\end{aligned}
$$

By construction,

$$
\mathcal{I}_{2} \leq \sigma\left|E_{k i}(h)\right| .
$$

Summing up over all the sets $E_{k i(h)}, k=1, \cdots, N(h), i=1, \cdots,\left|\mathcal{T}_{h}\right|$, we obtain for $\mathcal{I}_{1}$ :

$$
\begin{aligned}
\sum_{k=1}^{N(h)} \sum_{i=1}^{\left|\mathcal{T}_{h}\right|} \int_{E_{k i}(h)}\left|\mathcal{M}(u)-\mathcal{M}\left(\xi_{j}^{k i}\right)\right| \leq & \sum_{k=1}^{N(h)} \sum_{i=1}^{\left|\mathcal{T}_{h}\right|} \int_{E_{k i}(h)}\left|\mathcal{M}(u)-\mathcal{M}\left(U_{h}\right)\right| \\
& +\sum_{k=1}^{N(h)} \sum_{i=1}^{\left|\mathcal{T}_{h}\right|} \int_{E_{k i}(h)}\left|\mathcal{M}\left(U_{h}\right)-\mathcal{M}\left(\xi_{j}^{k i}\right)\right| .
\end{aligned}
$$

The first term tends to zero on account of the uniform $L^{\infty}\left(I ; H^{1,2}(\Omega)\right)$-property of $\left(U_{h}\right)_{h \rightarrow 0}$, Vitali's theorem and the strong convergence of $U_{h} \rightarrow u$ in $L^{2}\left(I ; C^{\beta}(\Omega)\right)$. To treat the second term, observe that $\xi_{j}^{k i} \in \operatorname{co}\left(U_{h}\left(k \tau, x_{0}\left(E_{i}\right)\right), \cdots, U_{h}\left(k \tau, x_{d}\left(E_{i}\right)\right)\right)$, and that for almost every $t \in I$, the discrete solution $U_{h}(t, \cdot)$ strongly converges to $u$ in $C^{\beta}(\Omega)$. As a consequence, $\sum_{k=1}^{N(h)} \sum_{i=1}^{\left|\mathcal{T}_{h}\right|} \xi_{j}^{k i} \cdot \chi_{E_{k i}(h)}(t, x)$ converges to $u(t, x)$ for arbitrary $x \in \Omega$. An application of Vitali's theorem shows that the second term converges to zero; hence the convergence of the field of discrete mobility matrices in $L^{1}\left(\Omega_{T}\right)$ is established. Another application of Vitali's theorem, combined with the uniform $L^{\infty}\left(I ; H^{1,2}(\Omega)\right)$-regularity of $U_{h}$ and Lemma 3.1, shows that the convergence in fact takes place in $L^{p}\left(\Omega_{T}\right)$ provided $p< \begin{cases}\infty & \text { if } d=2, \\ \frac{6}{n} & \text { if } d=3 .\end{cases}$

If $W^{\prime}$ is singular (e.g., if (H1) applies), the convergence of $\mathcal{I}_{h}\left(W_{+}^{\prime}\left(U_{h}(\cdot, \cdot)\right)+\right.$ $\left.W_{-}^{\prime}\left(U_{h}(\cdot-\tau, \cdot)\right)\right)$ to $W^{\prime}(u)$ is not a direct consequence of the $L^{2}\left(I ; C^{\beta}\right)$-convergence of $\left(U_{h}\right)_{h \rightarrow 0}$. Before formulating the result, it is worth reflecting upon the technical difficulties which will arise. If we only had to show that $W^{\prime}\left(U_{h}\right)$ converges to $W^{\prime}(u)$ on $S_{\delta}$ for $h \rightarrow 0$, the proof would be rather straightforward, using the convergence properties of $\left(U_{h}\right)_{h \rightarrow 0}$ and the equi-continuity of $W^{\prime}(\cdot)$ on $(\delta, \infty)$. But we need a corresponding result for $\mathcal{I}_{h} W^{\prime}\left(U_{h}\right)$, and in order to make use of the convergence properties of $W^{\prime}\left(U_{h}\right)$, it is necessary to control the norm

$$
\left\|\mathcal{I}_{h} W^{\prime}\left(U_{h}\right)-W^{\prime}\left(U_{h}\right)\right\|_{L^{2}\left(A_{h}\right)}
$$

uniformly for $h \rightarrow 0$ on sets $A_{h} \subset S_{\delta}$ satisfying $\lim _{h \rightarrow 0} A_{h}=S_{\delta}$ in an appropriate sense. This can be achieved, as the subsequent Lemma 8.7 shows, if we guarantee that $U_{h}$ is bounded from below by a positive number on these subsets $A_{h}$. That is the reason why we will confine ourselves in the following lemma to the convergence of $\mathcal{I}_{h} W^{\prime}\left(U_{h}\right) \chi_{\left[S_{\delta} \cap S_{\frac{\delta}{4}}^{\mathcal{T}_{h}}\right]}$. It reads as follows. 
Lemma 8.6. Let $\left(U_{h}\right)_{h \rightarrow 0}$ be a sequence of discrete solutions such that $\left(U_{h}(t-\tau, \cdot)\right)_{h \rightarrow 0}$ and $\left(U_{h}(t, \cdot)\right)_{h \rightarrow 0}$ uniformly converge to $u(t, \cdot)$ in $C^{\beta}(\Omega)$ for arbitrary $0<\beta<2-\frac{d}{2}$ and almost every $t \in I$. Then:

- $\mathcal{I}_{h} W_{+}^{\prime}\left(U_{h}\right) \chi_{\left[S_{\delta} \cap S_{\frac{\delta}{4}}^{\mathcal{T}_{h}}\right]}$ converges strongly to $W_{+}^{\prime}(u) \chi_{\left[S_{\delta}\right]}$ in $L^{2}\left(\Omega_{T}\right)$, and

- $\mathcal{I}_{h} W_{-}^{\prime}\left(U_{h}(\cdot-\tau)\right) \chi_{\left[S_{\frac{\delta}{4}}^{\mathcal{T}_{h}}\right]}(\cdot-\tau) \chi_{\left[S_{\delta}\right]}$ converges strongly to $W_{-}^{\prime}(u) \chi_{\left[S_{\delta}\right]}$ in $L^{2}\left(\Omega_{T}\right)$.

Remark. If $W_{+}^{\prime}(\cdot)$ (or $W_{-}^{\prime}(\cdot)$ ) is not singular at zero, then the stronger results $\mathcal{I}_{h} W_{+}^{\prime}\left(U_{h}\right) \rightarrow W_{+}^{\prime}(u)$ in $L^{2}\left(\Omega_{T}\right)\left(\right.$ or $\mathcal{I}_{h} W_{-}^{\prime}\left(U_{h}(\cdot-\tau, \cdot)\right) \rightarrow W_{-}^{\prime}(u)$ in $L^{2}\left(\Omega_{T}\right)$, respectively) can be obtained. On the other hand, if (H1) holds and $\varrho_{1}$ is sufficiently large, i.e.,

$$
\varrho_{1}> \begin{cases}4 & \text { if } d=2 \\ 9 & \text { if } d=3\end{cases}
$$

then $u(t, \cdot)$ is strictly positive for almost every $t \in(0, T)$, as a combination of the energy estimate and Corollary 6.2 shows. Hence, for almost every $t \in(0, T)$ and for sufficiently small $\delta>0$ the set $S_{\delta}(t)$ coincides with $\Omega$.

Proof. As a consequence of the uniform convergence of $\left(U_{h}(t-\tau, \cdot)\right)_{h \rightarrow 0}$ in $C^{\beta}(\Omega)$, the functions $\chi_{\left[S_{\frac{\delta}{4}}^{\tau_{h}}(t-\tau)\right]} \chi_{\left[S_{\delta}(t)\right]}$ converge strongly in $L^{2}(\Omega)$ to $\chi_{\left[S_{\delta}(t)\right]}$ for almost every $t \in I$. Therefore, also $W_{-}^{\prime}\left(U_{h}(t-\tau, \cdot)\right) \chi_{\left[S_{\frac{\delta}{4}}^{\mathcal{T}_{h}}(t-\tau)\right]} \chi_{\left[S_{\delta}(t)\right]}$ converges strongly to $W_{-}^{\prime}(u(t, \cdot)) \chi_{\left[S_{\delta}(t)\right]}$ in $L^{2}(\Omega)$ for almost every $t \in I$. Combining this result with the subsequent auxiliary Lemma8.7 and Lebesgue's theorem on majorized convergence, the assertion follows for $W_{-}^{\prime}$. The modifications necessary to treat $W_{+}^{\prime}$ are obvious.

It remains to prove the following auxiliary result

Lemma 8.7. Let $G: \mathbb{R} \rightarrow \mathbb{R}$ be of class $H^{1, \infty}$, let a regular triangulation $\mathcal{T}_{h}$ of a domain $\Omega$ be given which satisfies (T1), and let $V^{h}$ be the corresponding space of linear finite elements. Then, for arbitrary $U \in V_{h}$,

$$
\left\|\mathcal{I}_{h} G(U)-G(U)\right\|_{L^{2}(\Omega)} \leq C \cdot\|G\|_{1, \infty}^{2} \cdot h^{d} \int_{\Omega}|\nabla U|^{2}
$$

Proof. After an appropriate affine-equivalent transformation, we can assume an element $K$ to be given by

$$
K=\operatorname{co}\left(0, h \cdot s_{1} \cdot e_{1}, \cdots, h \cdot s_{d} \cdot e_{d}\right) .
$$

Here, $\left(e_{1}, \cdots, e_{d}\right)$ is the canonical basis of $\mathbb{R}^{d}$, and the positive numbers $\left(s_{i}\right)_{i=1, \cdots, d}$ are uniformly bounded away from zero and also bounded from above.

For arbitrary $x \in K$, we have

$$
G(U(x))=G(U(0))+G^{\prime}\left(\xi_{1}\right) \cdot\langle\nabla U, x\rangle
$$

with a number $\xi_{1} \in \operatorname{co}\left\{U_{0}, \cdots, U_{d}\right\}$. On the other hand,

$$
\left(\mathcal{I}_{h} G(U)\right)(x)=G\left(U_{0}\right)+\sum_{i=1}^{d}\left(x_{i} \cdot G^{\prime}\left(\zeta_{i}\right) \cdot \frac{U_{i}-U_{0}}{h \cdot s_{i}}\right)
$$

with numbers $\zeta_{i} \in \operatorname{co}\left\{U_{0}, \cdots, U_{d}\right\}$. 
Hence

$$
\left|G(U(x))-\left(\mathcal{I}_{h} G(U)\right)(x)\right| \leq d \cdot\left\|G^{\prime}\right\|_{L^{\infty}(\Omega)}|\nabla U| \cdot|x|
$$

Furthermore,

$$
\int_{K}\left|G(U(x))-\left(\mathcal{I}_{h} G(U)\right)(x)\right|^{2} \leq\left. C \cdot d^{2}\left\|G^{\prime}\right\|_{L^{\infty}(\Omega)}\left|\cdot h^{d} \int_{K}\right| \nabla U\right|^{2}
$$

which gives the result.

The main ingredient for the proof of Theorem 8.2 is the following lemma, which allows an identification of the limit $J$ of discrete fluxes $J_{h}$ with the continuous flux $\mathcal{M}(u) \nabla p$.

Lemma 8.8. Assume that (H1) or (H2) is satisfied and that a sequence $\left(U_{h}\right)_{h \rightarrow 0}$ of discrete solutions has the following properties:

$$
\begin{gathered}
U_{h} \rightarrow u \text { strongly in } L^{2}\left(I ; C^{\beta}(\Omega)\right) \text { for } 0<\beta<2-\frac{d}{2}, \\
-\Delta_{h} U_{h}=P_{h}-\mathcal{I}_{h} W_{+}^{\prime}\left(U_{h}\right)-\mathcal{I}_{h} W_{-}^{\prime}\left(U_{h}(\cdot-\tau, \cdot)\right) \rightarrow-\Delta u \\
\text { weakly in } L^{2}\left((\varepsilon, T) ; L^{2}(\Omega)\right) \text { for arbitrary } 0<\varepsilon<T,
\end{gathered}
$$

$J_{h}:=M_{\sigma}\left(U_{h}\right) \cdot \nabla P_{h} \rightarrow J$ weakly in $L^{2}\left(I ; L^{q_{1}}(\Omega)\right)$ for $q_{1}<\frac{4 d}{2 d+(d-2) n}$,

$$
\begin{gathered}
M_{\sigma}\left(U_{h}\right) \rightarrow \mathcal{M}(u) \text { strongly in } L^{p}\left(\Omega_{T}\right) \text { for all } p< \begin{cases}\infty & \text { if } d=2, \\
\frac{6}{n} & \text { if } d=3,\end{cases} \\
\mathcal{I}_{h} W_{+}^{\prime}\left(U_{h}\right) \chi_{\left[S_{\delta} \cap S_{\frac{\delta}{4}}^{\mathcal{T}_{h}}\right]} \rightarrow W_{+}^{\prime}(u) \chi_{\left[S_{\delta}\right]} \text { strongly in } L^{2}\left(\Omega_{T}\right), \\
\mathcal{I}_{h} W_{-}^{\prime}\left(U_{h}(\cdot-\tau)\right) \chi_{\left[S_{\frac{\delta}{4}}^{\mathcal{T}_{h}}\right]}(\cdot-\tau) \chi_{\left[S_{\delta}\right]} \rightarrow W_{-}^{\prime}(u) \chi_{\left[S_{\delta}\right]} \text { strongly in } L^{2}\left(\Omega_{T}\right) .
\end{gathered}
$$

Then a set $\mathcal{S} \subset I, \mu(\mathcal{S})=\mu(I)$, exists such that for all $t \in \mathcal{S}$, the function $u(t, \cdot)$ is Hölder-continuous to the exponent $\beta<2-\frac{d}{2}$, and a function $p(t, \cdot): \Omega \rightarrow \mathbb{R}$, $p(t, \cdot) \in H_{\mathrm{loc}}^{1}([u(t, \cdot)>0])$, exists such that

$$
\begin{aligned}
& p(t, \cdot)= \begin{cases}-\Delta u(t, \cdot)+W^{\prime}(u(t, \cdot)) & \text { on }[u(t, \cdot)>0], \\
0 & \text { on }[u(t, \cdot)=0],\end{cases} \\
& J(t, \cdot)= \begin{cases}\mathcal{M}(u(t, \cdot)) \nabla p(t, \cdot) & \text { on }[u(t, \cdot)>0], \\
0 & \text { on }[u(t, \cdot)=0] .\end{cases}
\end{aligned}
$$

Moreover, for arbitrary $\delta>0$ and $t \in \mathcal{S}$, the sequence $\left(P_{h}(t, \cdot)\right)_{h \rightarrow 0}$ converges weakly to $p(t, \cdot)$ in $H^{1}\left(S_{\delta}(t)\right)$.

Proof. Let us first prove the existence of a set $\mathcal{S} \subset I$ with $\mu(\mathcal{S})=\mu(I)$ such that for each $t \in \mathcal{S}$ and for a subsequence which we will denote again by the index $h$ we 
have

$\mathcal{I}_{h} W_{-}^{\prime}\left(U_{h}(t-\tau, \cdot)\right) \chi_{\left[S_{\frac{\delta}{4}}^{T_{h}}\right]}(t-\tau, \cdot) \chi_{\left[S_{\delta}\right]}(t, \cdot) \rightarrow W_{-}^{\prime}(u) \chi_{\left[S_{\delta}\right]}(t, \cdot)$ strongly in $L^{2}(\Omega)$,

$$
\begin{gathered}
M_{\sigma}\left(U_{h}(t, \cdot)\right) \rightarrow \mathcal{M}(u(t, \cdot)) \text { strongly in } L^{p}(\Omega) \text { for all } p<\frac{2 d}{(d-2) n}, \\
U_{h}(t, \cdot) \rightarrow u(t, \cdot) \text { strongly in } C^{\beta}(\Omega) \text { for all } \beta<2-\frac{d}{2}, \\
U_{h}(t-\tau, \cdot) \rightarrow u(t, \cdot) \text { strongly in } C^{\beta}(\Omega) \text { for all } \beta<2-\frac{d}{2}, \\
\mathcal{I}_{h} W_{+}^{\prime}\left(U_{h}\right) \chi_{\left[S_{\delta} \cap S_{\frac{\delta}{4}}^{\mathcal{T}_{h}}\right]}(t, \cdot) \rightarrow W_{+}^{\prime}(u) \chi_{\left[S_{\delta}\right]}(t, \cdot) \text { strongly in } L^{2}(\Omega),
\end{gathered}
$$

Let us first discuss (61)- (65). The existence of a set $\mathcal{S}_{1} \subset I$ with measure $\mu_{1}\left(\mathcal{S}_{1}\right)=$ $\mu_{1}(I)$ on which (61) - 65) are satisfied follows at once due to Corollary 8.4 and the fact that convergence in $L^{p}$ implies convergence almost everywhere for a subsequence. To prove a uniform bound on $\left(M_{\sigma}\left(U_{h}(t, \cdot)\right) \nabla P_{h}, \nabla P_{h}\right)$ and $\int_{\Omega}\left|\Delta U_{h}(t, \cdot)\right|^{2}$ for almost every $t \in I$, we argue as follows: Consider the set

$$
\mathcal{E}:=\left\{t \in I \mid \liminf _{h \rightarrow 0}\left(M_{\sigma}\left(U_{h}(t, \cdot)\right) \nabla P_{h}(t, \cdot), \nabla P_{h}(t, \cdot)\right)=+\infty\right\} .
$$

Define $K_{h}(t):=\left(M_{\sigma}\left(U_{h}(t, \cdot)\right) \nabla P_{h}(t, \cdot), \nabla P_{h}(t, \cdot)\right)$ and

$$
[A]_{L}:= \begin{cases}A & \text { if } A<L, \\ L & \text { otherwise }\end{cases}
$$

By the energy estimate,

$$
C \geq \int_{\mathcal{E}}\left(M_{\sigma}\left(U_{h}\right) \nabla P_{h}, \nabla P_{h}\right) \geq \int_{\mathcal{E}}\left[K_{h}(t)\right]_{L} d t \stackrel{h \rightarrow 0}{\longrightarrow} L \cdot|\mathcal{E}| .
$$

We let $L$ tend to infinity, and (67) is proven for almost every $t \in I$. A similar reasoning also applies to (66). Therefore a subset $\mathcal{S}$ with the required properties exists.

Let us next prove a result on weak convergence of $\Delta_{h} U_{h}(t, \cdot)$ in $\Omega$ pointwise for all $t \in \mathcal{S}$. Combining (62) and (66), we observe that the whole sequence $\left(\Delta_{h} U_{h}(t, \cdot)\right)_{h \rightarrow 0}$ converges to $\Delta u(t, \cdot)$ weakly in $L^{2}(\Omega)$ for $t \in \mathcal{S}$. This allows us to define for $t \in \mathcal{S}$ a function

$$
p(t, x):= \begin{cases}-\Delta u(t, x)+W^{\prime}(u(t, x)) & \text { if } u(t, x)>0, \\ 0 & \text { if } u(t, x)=0 .\end{cases}
$$

For $t \in \mathcal{S}$, the function $p(t, \cdot)$ is obviously an element of $L_{\text {loc }}^{2}(u(t, \cdot)>0)$, and on $S_{\delta}(t):=\{x \in \Omega \mid u(t, x) \geq \delta\}$ the function $p(t, \cdot)$ can be obtained as weak limit of the discrete pressure $P_{h}(t, \cdot)$. 
Let us now investigate for $\delta>0$ the limit behaviour of $\nabla P_{h}(t, \cdot)$ on $S_{\delta}(t)$ as $h$ tends to zero. We decompose

$$
P_{h}(t, \cdot)=\underbrace{-\Delta_{h} U_{h}(t, \cdot)+\mathcal{I}_{h} W_{+}^{\prime}\left(U_{h}(t, \cdot)\right)}_{P_{h}^{1}}+\underbrace{\mathcal{I}_{h} W_{-}^{\prime}\left(U_{h}(t-\tau, \cdot)\right)}_{P_{h}^{2}} .
$$

Relation (62) implies that

$$
S_{\delta}(t) \cap S_{\frac{\delta}{4}}^{\mathcal{T}_{h}}(t)=S_{\delta}(t)
$$

for $t \in \mathcal{S}$ and $h=h(t)$ sufficiently small. Take $\eta \in C_{0}^{\infty}\left(\left(S_{\delta}(t)\right)^{\circ} ; \mathbb{R}^{d}\right)$ and observe that $\int_{S_{\delta}(t)}\left|\nabla P_{h}(t, \cdot)\right|^{2}$ is uniformly bounded due to (67). We obtain, for a weak limit $\xi$,

$$
\int_{S_{\delta}(t)} \xi \cdot \eta=\lim _{h \rightarrow 0}\left\{-\int_{S_{\delta}(t)} P_{h}^{1} \operatorname{div} \eta-\int_{S_{\delta}(t)} P_{h}^{2} \operatorname{div} \eta\right\},
$$

provided the limit on the right-hand side exists. To prove this, let us first study the convergence behaviour of the first term on the right-hand side. By (62) and (64),

$$
\int_{S_{\delta}(t)} P_{h}^{1} \operatorname{div} \eta \stackrel{h \rightarrow 0}{\longrightarrow} \int_{S_{\delta}(t)}\left(-\Delta u(t, \cdot)+W_{+}^{\prime}(u(t, \cdot))\right) \operatorname{div} \eta
$$

for all $\eta \in C_{0}^{\infty}\left(\left(S_{\delta}(t)\right)^{\circ} ; \mathbb{R}^{d}\right)$. To show that $\int_{S_{\delta}(t)} P_{h}^{2} \operatorname{div} \eta$ converges for $h \rightarrow 0$ to $\int_{S_{\delta}(t)} W_{-}^{\prime}(u(t, \cdot)) \operatorname{div} \eta$, observe that (63) implies that

$$
S_{\delta}(t) \cap S_{\frac{\delta}{4}}^{\mathcal{T}_{h}}(t-\tau)=S_{\delta}(t)
$$

for $t \in \mathcal{S}$ and $h=h(t)$ sufficiently small. The previous argument can be repeated, and altogether we may identify

$$
\xi=\nabla p \text { on } S_{\delta}(t) .
$$

It remains to prove the identification

$$
J(t, \cdot)= \begin{cases}\mathcal{M}(u(t, \cdot) \nabla p(t, \cdot) & \text { on }[u(t, \cdot)>0], \\ 0 & \text { on }[u(t, \cdot)=0],\end{cases}
$$

for $t \in \mathcal{S}$.

Assumption (55) combined with (61), (67) and the fact that $\nabla P_{h}(t, \cdot)$ weakly converges on $S_{\delta}(t)$ to $\nabla p(t, \cdot)$ implies that

$$
J_{h}(t, \cdot) \rightarrow \mathcal{M}(u(t, \cdot)) \nabla p(t, \cdot)
$$

weakly in $L^{q}(\Omega)$ for all $q<\frac{4 d}{2 d+(d-2) n}$. The asserted identification on $[u(t, \cdot)>0]$ follows.

It remains to consider the set $\mathcal{N}=[u(t, \cdot)]=0$ for $t \in \mathcal{S}$. We have

$$
\begin{aligned}
\int_{\mathcal{N}} M_{\sigma}\left(U_{h}\right)\left|\nabla P_{h}\right|(t, \cdot) & \leq\left(\int_{\Omega} M_{\sigma}\left(U_{h}\right)\left|\nabla P_{h}\right|^{2}(t, \cdot)\right)^{\frac{1}{2}} \cdot\left(\int_{\mathcal{N}}\left|M_{\sigma}\left(U_{h}\right)\right|(t, \cdot)\right)^{\frac{1}{2}} \\
& =o_{h}(1)
\end{aligned}
$$

This proves the lemma.

Proof of Theorem 8.2. This is a direct consequence of Lemmas 8.3, 8.6, and 8.8

Passing to the limit as $h \rightarrow 0$ in the integral estimates, we obtain the following positivity result on the continuous solution $u$ : 
Theorem 8.9. For arbitrary $T>0$ there exists a positive constant $C(T)$ such that the solution u constructed in Theorem 8.2 satisfies the following integral estimate:

$$
\text { ess } \sup _{0<t<T}\left\{\int_{\Omega}|\nabla u|^{2}+\int_{\Omega} W(u)+\int_{\Omega} G(u)\right\}<C(T) .
$$

If (H1) holds or if $n>2$, then for almost every $t \in(0, T)$ the d-dimensional measure of the set $[u(t, \cdot)=0]$ vanishes.

Proof. Estimate (69) is a direct consequence of Fatou's lemma, the energy estimate (18), the entropy estimate (20) and the fact that $U_{h}(t, \cdot) \longrightarrow u(t, \cdot)$ in $C^{\beta}(\Omega)$, $0<\beta<2-\frac{d}{2}$, for almost every $t \in(0, T)$.

The asserted positivity result follows by the growth assumption on $W$ or the singular behaviour of $G(\cdot)$ near zero for $n>2$, respectively.

\section{Appendix}

To give a first flavor of the practicality of the numerical method analyzed in this paper, we present a simulation of film instabilities due to gravity effects (RayleighTaylor effect). From every day experience (for instance, a liquid film hanging below the ceiling), we expect the film to be unstable and we expect droplets to form. This effect is shown in Figure 1, where the different stages correspond to times $t_{1}=0.0, t_{2}=0.00289, t_{3}=0.00312$, and $t_{4}=0.005$. Note that the droplets are paraboloid-shaped, which is an effect due to the linearized curvature term in the lubrication model.

This simulation was performed together with Martin Rumpf, based on a joint numerical code. For more detail and simulations addressing different phenomena encountered in thin film flow, we refer to the papers [4] and [20].

Let us nevertheless make a few comments on the computational aspects. Choosing the domain $\Omega=(-1,1)^{2}$, we solve the equation

$$
u_{t}+\operatorname{div}\left(u^{2} \nabla(\Delta u+200 u)\right)=0
$$

using a simplicial triangulation with $128^{2}$ grid points. Note that the pressure $p=$ $-\Delta u-200 u$ is given as the sum of a linearized surface tension term and an additional gravity term which causes the instability.

As initial data, we take the interpolant of the function

$$
u_{0}(x, y)=\left(1+0.005 \sin \left(50(x-0.4)^{2}\right)\right)\left(1+0.0005 \sin \left(50(y+0.4)^{2}\right)\right) .
$$

We choose $\sigma=10^{-12}$ and use a time-step control inspired by the concept presented in [18. More precisely, we determine in each time-step $t_{k}$ on each element $E \in \mathcal{T}_{h}$ numbers

$$
v\left(t_{k}, E\right):= \begin{cases}\frac{\left.\mathcal{M}\left(\overline{\left(\overline{U_{\tau h}^{\sigma}}\right.}\right)_{E}\right)}{\left(\overline{U_{\tau h}^{\sigma}}\right)_{E}} \sum_{i=1}^{d}\left|\partial_{x_{i}} P_{\tau h}^{\sigma}\right| & \text { if }\left.U_{\tau h}^{\sigma}\right|_{E} \geq 0 \text { and }\left(\overline{U_{\tau h}^{\sigma}}\right)_{E}>0, \\ 0 & \text { otherwise }\end{cases}
$$

and we define the time-increment $\tau_{k}$ by the formula

$$
\tau_{k}:=\frac{\gamma h}{\beta+\max _{E \in \mathcal{T}_{h}} v\left(t_{k}, E\right)} \quad \text { with } 0<\beta<\gamma \leq 1 .
$$

The reader familiar with the modeling aspects of lubrication approximation will realize that the heuristics of that strategy are the following. Vertical averages of horizontal components of the fluid's velocity field correspond to the quantity 

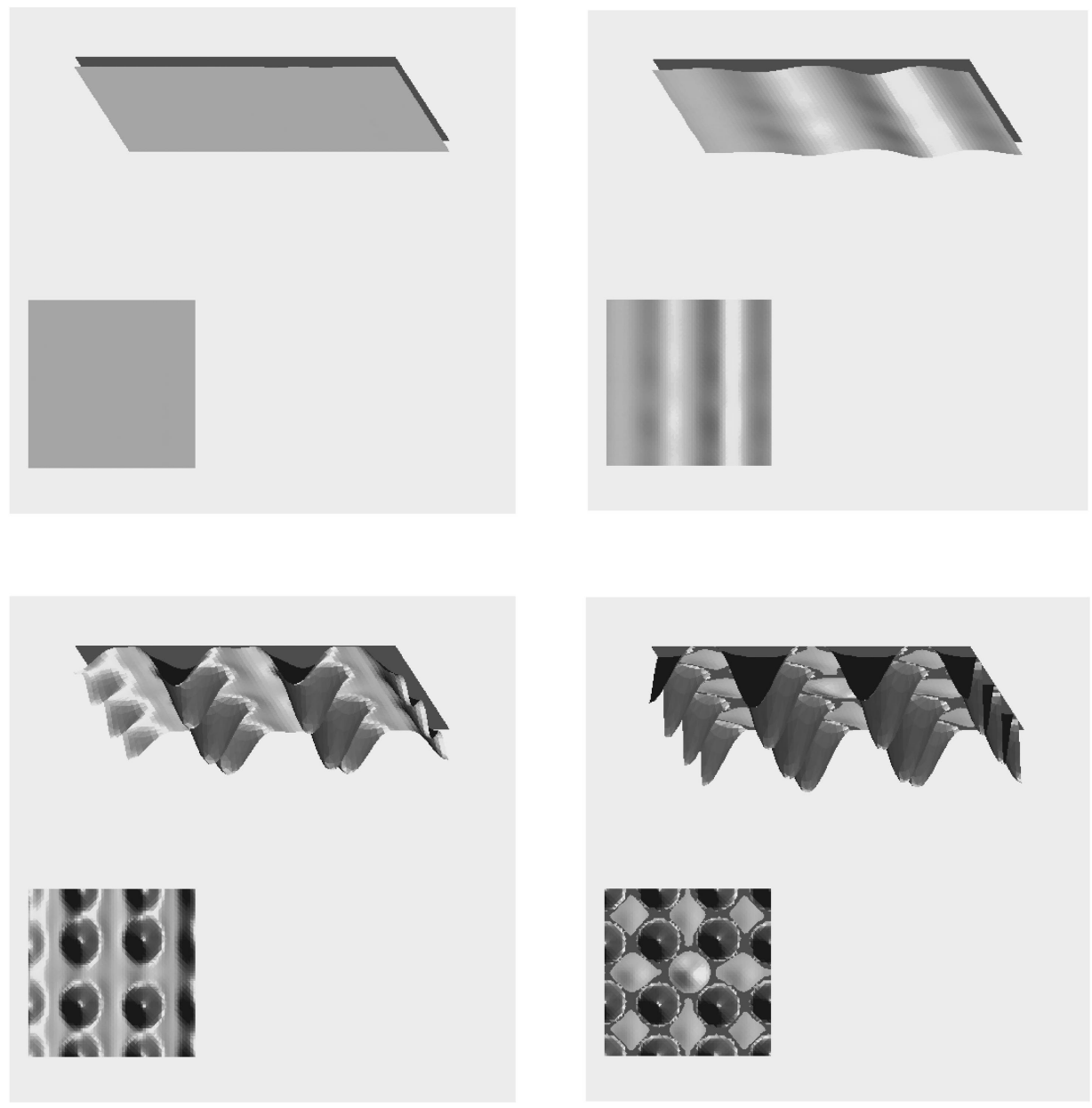

FiguRE 1. Droplet formation due to gravity effects - side view and bird's eye perspective.

$\frac{\mathcal{M}(u)}{u} \nabla p$, and as soon as these components become large, the time-increment is chosen small. Otherwise, large increments are allowed. This concept is not only well-suited to track contact lines efficiently (see [18]); in addition it also helps to reduce the number of iterations necessary to solve the arising discrete equations.

With respect to the analytical issues raised in the present paper, we note thatobviously-hypothesis (H2) is satisfied. Hence, discrete nonnegativity follows by the entropy estimate.

\section{ACKNOWLEDGMENT}

For the numerical example presented in the appendix, a numerical code developed together with Martin Rumpf has been used. 


\section{REFERENCES}

[1] J. Barrett, J. Blowey, and H. Garcke. Finite element approximation of a fourth order nonlinear degenerate parabolic equation. Numer. Math., 80:525-556, 1998. MR 99j:64144

[2] J. Barrett, J. Blowey, and H. Garcke. Finite element approximation of the Cahn-Hilliard equation with degenerate mobility. SIAM J. Num. Analysis, 37:286-318, 1999. MR 2001c:65118

[3] J. Barrett, J. Blowey, and H. Garcke. On fully practical finite element approximations of degenerate Cahn-Hilliard systems. Math. Model. Numer. Anal., 35:713-748, 2001.

[4] J. Becker, G. Grün, R. Seemann, H. Mantz, K. Jacobs, K.R. Mecke, and R. Blossey. Complex dewetting scenarios captured by thin film models. Nature Materials. In press.

[5] E. Beretta, M. Bertsch, and R. Dal Passo. Nonnegative solutions of a fourth order nonlinear degenerate parabolic equation. Arch. Ration. Mech. Anal., 129:175-200, 1995. MR 96b:35116

[6] F. Bernis. Viscous flows, fourth order nonlinear degenerate parabolic equations and singular elliptic problems. In J.I. Diaz, M.A. Herrero, A. Linan, and J.L. Vazquez, editors, Free boundary problems: theory and applications, Pitman Research Notes in Mathematics 323, pages 40-56. Longman, Harlow, 1995. MR 96b:00022

[7] MR 97e:35095 F. Bernis. Finite speed of propagation and continuity of the interface for thin viscous flows. Adv. Differential Equations, 1, no. 3:337-368, 1996. MR 97e:35095

[8] F. Bernis and A. Friedman. Higher order nonlinear degenerate parabolic equations. J. Differential Equations, 83:179-206, 1990. MR 91c:35078

[9] A.L. Bertozzi and M. Pugh. The lubrication approximation for thin viscous films: regularity and long time behaviour of weak solutions. Comm. Pure Appl. Math., 49(2):85-123, 1996. MR 97b:35114

[10] M. Bertsch, R. Dal Passo, H. Garcke, and G. Grün. The thin viscous flow equation in higher space dimensions. Adv. Differential Equations, 3:417-440, 1998. MR 2001a:35082

[11] Ph. G. Ciarlet. The finite element method for elliptic problems. North Holland, Amsterdam, 1978. MR 58:25001

[12] R. Dal Passo, H. Garcke, and G. Grün. On a fourth order degenerate parabolic equation: global entropy estimates and qualitative behaviour of solutions. SIAM J. Math. Anal., 29:321342, 1998. MR 99c:35118

[13] R. Dal Passo, L. Giacomelli, and G. Grün. A waiting time phenomenon for thin film equations. Ann. Scuola Norm. Sup. Pisa, XXX:437-463, 2001. CMP 2002:11

[14] C.M. Elliott and H. Garcke. On the Cahn-Hilliard equation with degenerate mobility. SIAM J. Math.Anal., 27 Nr. 2:404-423, 1996. MR 97c:35081

[15] C.M. Elliott and A.-M. Stuart. The global dynamics of discrete semilinear parabolic equations. Siam J. Numer. Anal., 30:1622-1663, 1993. MR 94j:65127

[16] G. Grün. Degenerate parabolic equations of fourth order and a plasticity model with nonlocal hardening. Z. Anal. Anwendungen, 14:541-573, 1995. MR 96m:35182

[17] G. Grün. On the numerical simulation of wetting phenomena. In W. Hackbusch and S. Sauter, editors, Proceedings of the 15th GAMM-Seminar Kiel, Numerical methods of composite materials. Vieweg-Verlag, Braunschweig. To appear.

[18] G. Grün and M. Rumpf. Nonnegativity preserving convergent schemes for the thin film equation. Num. Math., 87:113-152, 2000. MR 2002h:76108

[19] G. Grün and M. Rumpf. Simulation of singularities and instabilities in thin film flow. Euro. J. Appl. Math., 12:293-320, 2001.

[20] C. Neto, K. Jacobs, R. Seemann, R. Blossey, J. Becker, and G. Grün. Satellite hole formation during dewetting: experiment and simulation. Submitted for publication.

[21] A. Oron, S.H. Davis, and S.G. Bankoff. Long-scale evolution of thin liquid films. Reviews of Modern Physics, 69:932-977, 1997.

[22] K. Yosida. Functional analysis. Springer-Verlag, 1971. MR 50:2851]

[23] L. Zhornitskaya and A.L. Bertozzi. Positivity preserving numerical schemes for lubricationtype equations. SIAM J. Num. Anal., 37:523-555, 2000. MR 2000m:65100

Universität Bonn, Institut für Angewandte Mathematik, Beringstr. 6, 53115 Bonn, Germany

E-mail address: gg@iam.uni-bonn.de 